\title{
Optimal control of a fractional diffusion Sturm-Liouville problem on a star graph
}

\section{Contrôle optimal d'une diffusion fractionnée Problème de Sturm-Liouville sur un graphe étoile}

\author{
Pasquini Soh Fotsing \\ University of Buea, Department of Mathematics, Buea, Cameroon \\ pasquini.soh@aims-cameroon.org
}

ABSTRACT. This paper is devoted to parabolic fractional boundary value problems involving fractional derivative of Sturm-Liouville type. We investigate the existence and uniqueness results on an open bounded real interval, prove the existence of solutions to a quadratic boundary optimal control problem and provide a characterization via optimality system. We then investigate the analogous problems for a parabolic fractional Sturm-Liouville problem on a star graph with mixed Dirichlet and Neumann boundary controls.

AMS Subject Classification. 35J20,49J45,49J20.

KEYWORDS. Rieman-Liouville fractional derivative, Caputo fractional derivative, fractional integral, Sturm-Liouville equations, boundary value problem, optimal control, optimality system.

\section{Introduction}

Differential equation of fractional order has been used to to describe different processes in various fields such as optimisation, finance, continuum mechanics, quantum physics and etc. The motivation for the growing interest in studying such a problems (fractional boundary value problems) lies in one hand on the large number of possible applications in modeling of several complex natural phenomena for which the integer order of derivative approach turn up to be inappropriate. In some cases such as anomalous transport and diffusion, the classical approach is limited. On the other side, the concepts of fractional derivatives has emerged as it proved to describe some phenomena more accurately than the integer order derivatives.

A graph $G=(V, E)$ consists of a finite set $V=\left\{v_{1}, v_{2}, \cdots, v_{k}\right\}$ of vertices or nodes in a connected graph $G$ and a set $E=\left\{e_{1}, e_{2}, \cdots, e_{k}\right\}$ of edges. The distance between every two vertices $u, v \in V$ is minimum number of edges onto a $u v$-path of $G$. A differential equation on a graph is a collection of differential equations on the individual edges of $G$ based on the local coordinate systems (assigned each edge with the origin at one of the nodes). The theory of differential equation on graphs originated in 1980s with the work of Lumer in the framework of ramification spaces [19]. [19]. Many researchers have focused their attention on fractional differential equations (FDEs) problems which are actually obtained by replacing the ordinary derivatives by fractional derivatives. For instance, In [11], D. Idczak et al. used Riemann-Liouville fractional derivative to introduce and characterize fractional Sobolev space. He then gave some imbedding results and applied the obtained results to Sturm-Liouville fractional equations expressed in term of composition of right and left Riemann-Liouville fractional derivatives. In [1], G. Mophou et al. studies the optimal boundary control of a fractional Sturm-Liouville problem on the star graph. M. Klimek et al. [12] applied variational methods to prove the existence of a countable set of orthogonal solutions and corresponding eigenvalues to a fractional Sturm-Liouville equation. The Sturm-Liouville operator considered was a composition of right and left fractional Riemann-Liouville 
derivatives. Torebek et al. [14] studied a symmetric fractional Sturm-Liouville operator composed of Left Caputo and Right Riemann-Liouville fractional derivatives in a suitable Hilbert space and derived some spectral properties. In [13], Y. Liu et al. studied the existence of solutions to a nonlinear fractional equation involving a Sturm-Liouville operator obtained as a composition of two left Riemann-Liouville fractional derivatives. We also refer to $[15,16,37]$ and the reference therein for more literature on fractional Sturm-Liouville equations. As for the fractional equation on network few are done. In [18], O.Kh. Abdullaev et al. investigate a boundary value-problem for a fractional diffusion equations on a metric graphs involving fractional Caputo derivatives. Using the method of separation of variable, they obtained an existence results. V. Mehandiratta et al. [19] Studied the existence and uniqueness of a nonlinear Caputo fractional boundary value problem on a star graph. The results is obtained by means of Banach's contraction principle and Schaefer's fixed point theorem.

In this paper, we are interested in the optimal control of fractional diffusion Sturm-Liouville boundary value problems on a star graph:

$$
\begin{array}{ll}
\partial_{t} y^{i}+\mathcal{D}_{b_{i}^{-}}^{\alpha}\left(\beta^{i} \mathbb{D}_{a^{+}}^{\alpha} y^{i}\right)+q^{i} y^{i} & =f^{i}, \text { in }\left(a, b_{i}\right) \times(0, T), i=1, \ldots, n, \\
I_{a^{+}}^{1-\alpha} y^{i}\left(a^{+}\right) & =I_{a^{+}}^{1-\alpha} y^{j}\left(a^{+}\right) \text {in }(0, T), i \neq j=1, \ldots, n, \\
\sum_{i=1}^{n} \beta^{i}(a) \mathbb{D}_{a^{+}}^{\alpha} y^{i}\left(a^{+}\right) & =0, \quad \text { in }(0, T), \\
I_{a^{+}}^{1-\alpha} y^{1}\left(b_{1}^{-}\right) & =0, \quad \text { in }(0, T), \\
I_{a^{+}}^{1-\alpha} y^{i}\left(b_{i}^{-}\right) & =v_{i}, \quad \text { in }(0, T), i=2, \ldots, m \\
\beta^{i}\left(b_{i}\right) \mathbb{D}_{a^{+}}^{\alpha} y^{i}\left(b_{i}^{-}\right) & =v_{i}, \text { in }(0, T), i=m+1, \ldots, n, \\
y^{i}(0) & =y^{0, i}, \text { in }\left(a, b_{i}\right), \quad i=1, \ldots, n .
\end{array}
$$

where $\mathbb{D}_{a^{+}}^{\alpha}$ and $\mathcal{D}_{b_{i}^{-}}^{\alpha}, i=1, \ldots, n$ stand, respectively, for the left Riemann Liouville and the right Caputo fractional derivative of order $\alpha \in(0,1)$, respectively. $I_{a^{+}}^{\alpha}$ is the Riemann-Liouville fractional integral of order $\alpha$. The real functions $\beta^{i}$ and $q^{i} i=1, \ldots, n$ are defined on $\left[a, b_{i}\right], i=1, \ldots, n$ and satisfy suitable conditions to be discussed below, the function $f^{i}$ belongs to $L^{2}\left(a, b_{i}\right), i=1, \ldots, n$ and the controls $v_{i}, i=2, \ldots, n$ are real variables. Clearly, some controls can be equal to zero. The setting should, therefore, indicate that we are looking at a star graph, rooted at $b_{1}^{-}$, i.e., where we have a fixed Dirichlettype boundary condition and controlled via fractional Dirichlet and Neumann boundary conditions [see figure 1].

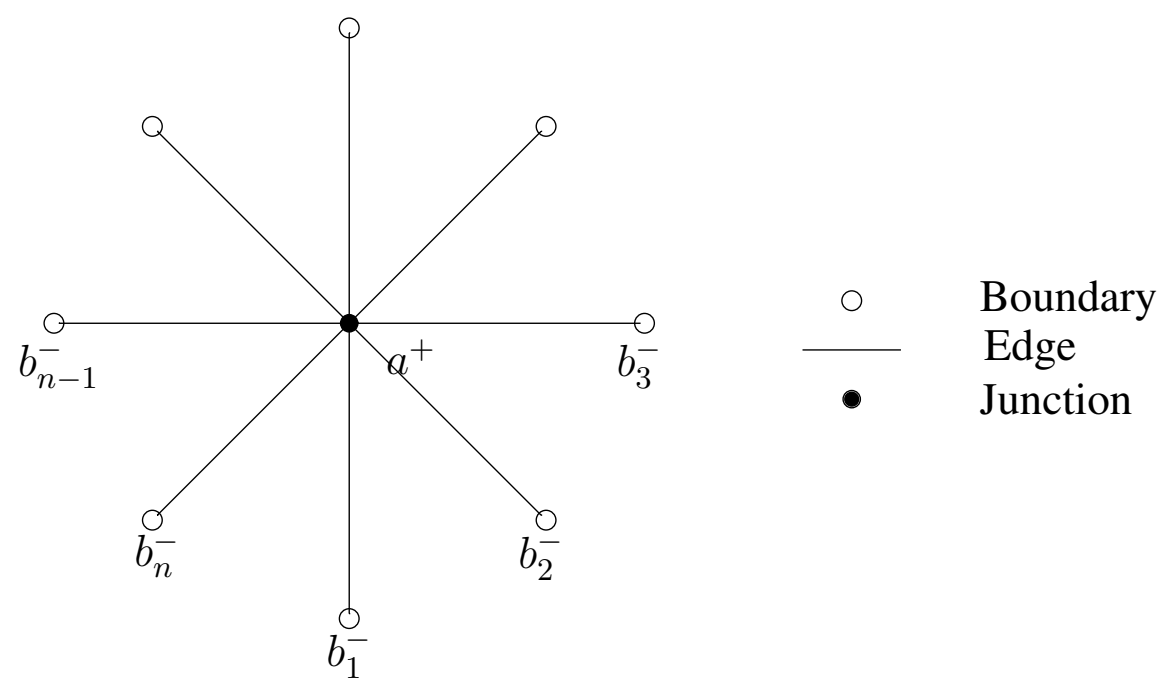

Figure 1. A sketch of the star graph with $n$ edges. 
In order to get started, we first consider the mixed problem on a single edge

$$
\left\{\begin{array}{llll}
\partial_{t} y+\mathcal{D}_{b^{-}}^{\alpha}\left(\beta \mathbb{D}_{a^{+}}^{\alpha}\right)(y)+q y & =f & \text { in }(a, b) \times(0, T) \\
\left(I_{a^{+}}^{1-\alpha} y\right)\left(a^{+}\right) & =0 & \text { in }(0, T) \\
\left(\beta \mathbb{D}_{a^{+}}^{\alpha} y\right)\left(b^{-}\right) & =v & \text { in }(0, T) \\
y(0) & =y^{0} & \text { in }(a, b)
\end{array}\right.
$$

Where $\mathcal{D}_{b^{-}}^{\alpha}$ and $\mathbb{D}_{a^{+}}^{\alpha}$ stand respectively for the right Caputo and left Riemann-Liouville fractional derivative of order $\alpha \in(0,1)$. The real function $\beta$ and $q$ are defined on $[a, b]$, and satisfy suitable conditions to be discussed below. The function $f$ belongs to $L^{2}(a, b)$ and $v$ is a real variable known as control.

The rest of this paper is organized as follows. In Section 2, we defined some notions on fractional calculus and some useful lemmas. Section 3 is devoted to the study of the optimal control problem on a single edge. The optimal control problem as well as his characterization by an optimality system on a star graph is investigate in Section 4. A conclusion is given in Section 5.

\section{Preliminaries}

In this section we give some definitions and some results useful for he study of the optimal control problems.

Definition 2.1. [20] Let $a \geq 0, f: \mathbb{R}_{+} \rightarrow \mathbb{R}$ and $\alpha>0$. Then the left and right Riemann-Liouville integral of order $\alpha$ of a function $f$ are respectively defined by

$$
\begin{aligned}
I_{a^{+}}^{\alpha}(f)(t) & =\frac{1}{\Gamma(\alpha)} \int_{a}^{t}(t-s)^{\alpha-1} f(s) d s, \\
I_{b^{-}}^{\alpha}(f)(t) & =\frac{1}{\Gamma(\alpha)} \int_{t}^{b}(s-t)^{\alpha-1} f(s) d s,
\end{aligned}
$$

provided that the integrals exist.

Definition 2.2. [21] Let $a \geq 0$ and $f: \mathbb{R}_{+} \rightarrow \mathbb{R}$. Then the left and right Riemann-Liouville fractional derivative of order $\alpha \in(0,1)$ of $f$ are defined respectively by:

$$
\begin{aligned}
& \mathbb{D}_{a^{+}}^{\alpha}(f)(t)=\frac{1}{\Gamma(1-\alpha)} \frac{d}{d t} \int_{a}^{t}(t-s)^{-\alpha} f(s) d s, \\
& \mathbb{D}_{b^{-}}^{\alpha}(f)(t)=\frac{-1}{\Gamma(1-\alpha)} \frac{d}{d t} \int_{b}^{t}(s-t)^{-\alpha} f(s) d s, \quad a<t<b,
\end{aligned}
$$

provided that the integrals exist. 
Definition 2.3. $[23,22]$ Let $a \geq 0$ and $f: \mathbb{R}_{+} \rightarrow \mathbb{R}$. Then the left and right Caputo fractional derivative of order $\alpha \in(0,1)$ of $f$ are defined respectively by:

$$
\begin{aligned}
\mathcal{D}_{a^{+}}^{\alpha} f(t) & =\frac{1}{\Gamma(1-\alpha)} \int_{a}^{t}(t-s)^{-\alpha} f^{\prime}(s) d s, \\
\mathcal{D}_{b^{-}}^{\alpha} f(t) & =\frac{-1}{\Gamma(1-\alpha)} \int_{t}^{b}(s-t)^{-\alpha} f^{\prime}(s) d s, \quad a<t<b,
\end{aligned}
$$

provided that the integrals exist.

From the definitions above, we have the following

$$
\begin{aligned}
I_{a^{+}}^{\alpha} \mathcal{D}_{a^{+}}^{\alpha}(f)(t) & =f(t)-f(a), \\
I_{a^{+}}^{\alpha} \mathbb{D}_{a^{+}}^{\alpha}(f)(t) & =f(t) .
\end{aligned}
$$

From the proof of Theorem 2.6 in [21], we have the following results.

Lemma 2.1. Let $\alpha>0$ and $\rho:(a, b) \rightarrow \mathbb{R}$ such that $\rho \in L^{2}(a, b)$. Then

$$
\begin{aligned}
\left\|I_{a^{+}}^{\alpha} \rho\right\|_{L^{2}(a, b)} & \leq \frac{(b-a)^{\alpha}}{\alpha \Gamma(\alpha)}\|\rho\|_{L^{2}(a, b)} \\
\left\|I_{b^{-}}^{\alpha} \rho\right\|_{L^{2}(a, b)} & \leq \frac{(b-a)^{\alpha}}{\alpha \Gamma(\alpha)}\|\rho\|_{L^{2}(a, b)} .
\end{aligned}
$$

Now, let $c_{0}, d_{0} \in \mathbb{R}$. Let also $f:[a, b] \rightarrow \mathbb{R}$ with the representation

$$
f(t)=\frac{c_{0}}{\Gamma(\alpha)}(t-a)^{\alpha-1}+I_{a^{+}}^{\alpha} \varphi(t), \quad \forall t \in[a, b] a . e
$$

and $g:[a, b] \rightarrow \mathbb{R}$ with the representation

$$
g(t)=\frac{d_{0}}{\Gamma(\alpha)}(b-t)^{\alpha-1}+I_{b^{-}}^{\alpha} \psi(t), \quad \forall t \in[a, b] a . e,
$$

where $\varphi$ and $\psi$ belong to $L^{2}(a, b)$. We denote by $A C_{a^{+}}^{\alpha, 2}$ and $A C_{b^{-}}^{\alpha, 2}$ the space of all function $f$ and $g$ with representation (1) and (2) respectively with $\varphi \in L^{2}(a, b)$ and $\psi \in L^{2}(a, b)$.

Remark 1. We have

$$
\begin{aligned}
& \mathbb{D}_{a^{+}}^{\alpha} f \in L^{2}(a, b) \Leftrightarrow f \in A C_{a^{+}}^{\alpha, 2}, \\
& \mathbb{D}_{b^{-}}^{\alpha} f \in L^{2}(a, b) \Leftrightarrow f \in A C_{b^{-}}^{\alpha, 2}
\end{aligned}
$$

For the proof and more literature of such space we refer to [11]. Then we can easily compute the following results.

Set

$$
H_{a^{+}}^{\alpha}(a, b)=A C_{a^{+}}^{\alpha, 2} \cap L^{2}(a, b)
$$

and

$$
H_{b^{-}}^{\alpha}(a, b)=A C_{b^{-}}^{\alpha, 2} \cap L^{2}(a, b)
$$


Then it follows from the definition $A C_{a^{+}}^{\alpha, 2}$ and $A C_{b^{-}}^{\alpha, 2}$ that,

$$
\rho \in H_{a^{+}}^{\alpha}(a, b) \Rightarrow \rho \in L^{2}(a, b) \text { and } \mathbb{D}_{a^{+}}^{\alpha} \rho \in L^{2}(a, b)
$$

and

$$
\rho \in H_{b^{-}}^{\alpha}(a, b) \Rightarrow \rho \in L^{2}(a, b) \text { and } \mathbb{D}_{b^{-}}^{\alpha} \rho \in L^{2}(a, b) .
$$

Then define on $H_{a^{+}}^{\alpha}(a, b)$, the inner product for all $\varphi, \psi \in H_{a^{+}}^{\alpha}(a, b)$ :

$$
(\varphi, \psi)_{H_{a^{+}}^{\alpha}(a, b)}=\int_{a}^{b} \varphi \psi d x+\int_{a}^{b} \mathbb{D}_{a^{+}}^{\alpha} \varphi \mathbb{D}_{a^{+}}^{\alpha} \phi d x .
$$

Lemma 2.2 ([11], Theorem 25). Then $H_{a^{+}}^{\alpha}(a, b)$ endowed with the norm

$$
\|\varphi\|_{H_{a^{+}}^{\alpha}(a, b)}^{2}=\|\varphi\|_{L^{2}(a, b)}^{2}+\left\|\mathbb{D}_{a^{+}}^{\alpha} \varphi\right\|_{L^{2}(a, b)}^{2}
$$

is a Hilbert space.

Set

$$
\mathcal{V}=\left\{y \in H_{a^{+}}^{\alpha}(a, b) \text { such that } \mid \mathcal{D}_{b^{-}}^{\alpha}\left(\beta \mathbb{D}_{a^{+}}^{\alpha} y\right) \in H_{b^{-}}^{1-\alpha}(a, b)\right\} .
$$

Then $\mathcal{V}$ is closed in $H_{a^{+}}^{\alpha}(a, b)$. Consequently, $\mathcal{V}$ endowed with norm (10) is a Hilbert space. The following trace results are useful for some calculation in the upcoming sections.

Lemma 2.3. Let $0<\alpha<1$ and $\gamma=1-\alpha$. Let also $\rho \in \mathcal{V}$. Then

1) The reals $I_{a^{+}}^{1-\alpha} \rho\left(a^{+}\right)$and $I_{a^{+}}^{1-\alpha} \rho\left(b^{-}\right)$exist.

2) The reals $\left(\beta \mathbb{D}_{a^{+}}^{\alpha} \rho\right)\left(a^{+}\right)$and $\left(\beta \mathbb{D}_{a^{+}}^{\alpha} \rho\right)\left(b^{-}\right)$exist.

\section{Proof.}

1) If $\rho \in \mathcal{V}$ then $\rho \in H_{a^{+}}^{\alpha}(a, b)$. thus, we have that $\rho \in L^{2}(a, b)$ and $\mathbb{D}_{a^{+}}^{\alpha} \rho=\frac{d}{d t} I_{a^{+}}^{1-\alpha} \rho \in L^{2}(a, b)$. But we also have $I_{a^{+}}^{1-\alpha} \rho \in L^{2}(a, b)$ because of Lemma 2.1 so that $I_{a^{+}}^{1-\alpha} \in H^{1}(a, b)$. Observing that the imbedding of $H^{1}(a, b)$ into $C([a, b])$ is compact, we have that $I_{a^{+}}^{1-\alpha} \rho\left(a^{+}\right)$and $I_{a^{+}}^{1-\alpha} \rho\left(b^{-}\right)$exist and belong to $\mathbb{R}$.

2) If $\rho \in \mathcal{V}$ then $\mathcal{D}_{b^{-}}^{\alpha}\left(\beta \mathbb{D}_{a^{+}}^{\alpha} \rho\right) \in H_{b^{-}}^{\gamma}(a, b)$. Thus we have

$$
\mathcal{D}_{b^{-}}^{\alpha}\left(\beta \mathbb{D}_{a^{+}}^{\alpha} \rho\right) \in L^{2}(a, b)
$$

and

$$
\mathbb{D}_{b^{-}}^{\gamma}\left(\mathcal{D}_{b^{-}}^{\alpha}\left(\beta \mathbb{D}_{a^{+}}^{\alpha} \rho\right)\right) \in L^{2}(a, b) .
$$

Observing that

$$
\begin{aligned}
\mathcal{D}_{b^{-}}^{\alpha}\left(\beta \mathbb{D}_{a^{+}}^{\alpha} \rho\right)(t) & =I_{b^{-}}^{1-\alpha}\left(\frac{d}{d t}\left(\beta \mathbb{D}_{a^{+}}^{\alpha} \rho\right)(t)\right) \\
& =I_{b^{-}}^{\gamma}\left(\frac{d}{d t}\left(\beta \mathbb{D}_{a^{+}}^{\alpha} \rho\right)(t)\right),
\end{aligned}
$$


we have that

$$
\begin{aligned}
\mathbb{D}_{b^{-}}^{\gamma}\left(\mathcal{D}_{b^{-}}^{\alpha}\left(\beta \mathbb{D}_{a^{+}}^{\alpha} \rho\right)\right)(t) & =\mathbb{D}_{b^{-}}^{\gamma} I_{b^{-}}^{\gamma}\left(\frac{d}{d t}\left(\beta \mathbb{D}_{a^{+}}^{\alpha} \rho\right)(t)\right) \\
& =\frac{d}{d t}\left(\beta \mathbb{D}_{a^{+}}^{\alpha} \rho\right)(t)
\end{aligned}
$$

which in view of (13) implies that

$$
\frac{d}{d t}\left(\beta \mathbb{D}_{a^{+}}^{\alpha} \rho\right) \in L^{2}(a, b)
$$

But we also have $\beta \mathbb{D}_{a^{+}}^{\alpha} \rho \in L^{2}(a, b)$ because $\rho \in H_{a^{+}}^{\alpha}(a, b)$ and $\beta \in \mathcal{C}([a, b])$. Consequently, $\beta \mathbb{D}_{a^{+}}^{\alpha} \rho \in$ $H^{1}(a, b)$ which is compactly imbedded in $\mathcal{C}([a, b])$. Thus $\left(\beta \mathbb{D}_{a^{+}}^{\alpha} \rho\right)\left(a^{+}\right)$and $\left(\beta\left(\mathbb{D}_{a^{+}}^{\alpha} \rho\right)\left(b^{-}\right)\right.$exist and belong to $\mathbb{R}$.

Lemma 2.4. [[11], Theorem 24]. Let $0<\alpha \leq 1$. Then the norm defined on $H_{a^{+}}^{\alpha}(a, b)$ by (10) is equivalent to the norm

$$
\left\|\left|\varphi\left\|_{H_{a^{+}}^{\alpha}(a, b)}^{2}=\left|I_{a^{+}}^{1-\alpha} \varphi\left(a^{+}\right)\right|^{2}+\right\| \mathbb{D}_{a^{+}}^{\alpha} \varphi \|_{L^{2}(a, b)}^{2} .\right.\right.
$$

In other words there exists $C_{1}>0$ positif such that

$$
\|\varphi\|\left\|_{H_{a^{+}}^{\alpha}(a, b)} \leq C_{1}\right\| \varphi\left\|_{H_{a^{+}}^{\alpha}(a, b)} \leq\right\| \mid \varphi \|_{H_{a^{+}}^{\alpha}(a, b)}, \quad \forall \varphi \in H_{a^{+}}^{\alpha}(a, b)
$$

Then we can easily compute the following results concerning integration by parts.

Lemma 2.5. $y, \phi:[a, b] \rightarrow \mathbb{R}$ be such that $y, \phi \in \mathcal{V}$. Then

$$
\begin{aligned}
& \int_{a}^{b} \mathcal{D}_{b^{-}}^{\alpha} y(s) \phi(s) d s=-\left[y(s)\left(I_{a^{+}}^{1-\alpha} \phi\right)(s)\right]_{s=a}^{s=b}+\int_{a}^{b} y(s)\left(\mathbb{D}_{a^{+}}^{\alpha} \phi\right)(s) d s \\
& \int_{a}^{b}\left(\mathbb{D}_{a^{+}}^{\alpha} \phi\right)(s) y(s) d s=\left[\left(I_{a^{+}}^{1-\alpha} \phi\right)(s) y(s)\right]_{s=a}^{s=b}+\int_{a}^{b} \phi(t)\left(\mathcal{D}_{b^{-}}^{\alpha} y\right)(t) d t
\end{aligned}
$$

Lemma 2.6. Let $\beta \in \mathcal{C}([a, b])$. Let also $y, \phi:[a, b] \rightarrow \mathbb{R}$ be such that $y, \phi \in \mathcal{V}$. Then

$$
\begin{aligned}
\int_{a}^{b} \mathcal{D}_{b^{-}}^{\alpha}\left(\beta \mathbb{D}_{a^{+}}^{\alpha} y\right)(s) \phi(s) d s & =-\left[\left(\beta \mathbb{D}_{a^{+}}^{\alpha} y\right)(s) I_{a^{+}}^{1-\alpha}(\phi)(s)\right]_{s=a}^{s=b} \\
& \left.+\int_{a}^{b}\left(\beta \mathbb{D}_{a^{+}}^{\alpha} y\right)(s) \mathbb{D}_{a^{+}}^{\alpha}(\phi)\right)(s) d s \\
& =-\left[\left(\beta \mathbb{D}_{a^{+}}^{\alpha} y\right)(s) I_{a^{+}}^{1-\alpha}(\phi)(s)\right]_{s=a}^{s=b} \\
& +\left[I_{a^{+}}^{1-\alpha}(y)(s) \beta(s) \mathbb{D}_{a^{+}}^{\alpha}(\phi)(s)\right]_{s=a}^{s=b} \\
& +\int_{a}^{b} y(t) \mathcal{D}_{b^{-}}^{\alpha}\left(\beta \mathbb{D}_{a^{+}}^{\alpha}(\phi)\right)(t) d t
\end{aligned}
$$

Proof. This is straightforward from (16)and (17). 
Lemma 2.7. Let $\beta \in \mathcal{C}([a, b])$ such that $\beta>\beta_{0}>0$. Let also $q \in \mathcal{C}([a, b])$ such that $q>q_{0}>0$. For any $y, z \in H_{a^{+}}^{\alpha}(a, b)$, we define the bilinear functional $a(.,$.$) by:$

$$
a(y, z)=\int_{a}^{b} \beta(x) \mathbb{D}_{a^{+}}^{\alpha} y(x) \mathbb{D}_{a^{+}}^{\alpha} z(x) d x+\int_{a}^{b} q(x) y(x) z(x) d x .
$$

Then, $a(.,$.$) is continuous and coercive on H_{a^{+}}^{\alpha}(a, b) \times H_{a^{+}}^{\alpha}(a, b)$.

Proof. Let $y, z \in H_{a^{+}}^{\alpha}(a, b)$. Using the Cauchy-Schwarz inequality and the definition of the norm on $H_{a^{+}}^{\alpha}(a, b)$ given by (10), we have

$$
\begin{aligned}
& |a(y, z)|=\left|\int_{a}^{b} \beta(x) \mathbb{D}_{a^{+}}^{\alpha} y(x) \mathbb{D}_{a^{+}}^{\alpha} z(x) d x+\int_{a}^{b} q(x) y(x) z(x) d x\right| \leq \\
& \|\beta\|_{\infty}\left\|\mathbb{D}_{a^{+}}^{\alpha} y\right\|_{L^{2}(a, b)}\left\|\mathbb{D}_{a^{+}}^{\alpha} z\right\|_{L^{2}(a, b)}+\|q\|_{\infty}\|y\|_{L^{2}(a, b)}\|z\|_{L^{2}(a, b)} \leq \\
& \left(\|q\|_{\infty}+\|\beta\|_{\infty}\right)\left[\left\|\mathbb{D}_{a^{+}}^{\alpha} y\right\|_{L^{2}(a, b)}^{2}+\|y\|_{L^{2}(a, b)}^{2}\right]^{1 / 2}\left[\left\|\mathbb{D}_{a^{+}}^{\alpha} z\right\|_{L^{2}(a, b)}^{2}+\|z\|_{L^{2}(a, b)}^{2}\right]^{1 / 2} \\
& =\left(\|q\|_{\infty}+\|\beta\|_{\infty}\right)\|y\|_{H_{a^{+}}^{\alpha}(a, b)}\|z\|_{H_{a^{+}}^{\alpha}(a, b)},
\end{aligned}
$$

and

$$
\begin{aligned}
a(y, y) & =\int_{a}^{b} \beta(x)\left|\mathbb{D}_{a^{+}}^{\alpha} y(x)\right|^{2} d x+\int_{a}^{b} q(x)|y(x)|^{2} d x \\
& \geq \min \left(\beta_{0}, q_{0}\right)\|y\|_{H_{a^{+}}^{\alpha}(a, b)}^{2} .
\end{aligned}
$$

This means that $a(.,$.$) is, respectively, continuous and coercive on H_{a^{+}}^{\alpha}(a, b) \times H_{a^{+}}^{\alpha}(a, b)$.

We will also need the following results for the optimal control problems below.

Lemma 2.8. Let $0<\alpha<1$. Let also $\left(y_{n}\right)$ be such that $\left\|y_{n}\right\|_{L^{2}\left(0, T ; H_{a^{+}}^{\alpha}(a, b)\right)} \leq C$ for some $C>0$. Then there exists $C>0$ such that,

$$
\begin{aligned}
\left\|y_{n}\right\|_{L^{2}\left(0, T ; L^{2}(a, b)\right)} & \leq C, \\
\left\|\mathbb{D}_{a^{+}}^{\alpha} y_{n}\right\|_{L^{2}\left(0, T ; L^{2}(a, b)\right)} & \leq C, \\
\left\|I_{a^{+}}^{1-\alpha} y_{n}\right\|_{L^{2}\left(0, T ; L^{2}(a, b)\right)} & \leq C, \\
\left\|I_{a^{+}}^{1-\alpha} y_{n}\right\|_{H^{1}\left(0, T ; L^{2}(a, b)\right)} & \leq C .
\end{aligned}
$$

Proof. If $\left\|y_{n}\right\|_{L^{2}\left(0, T ; H_{a^{+}}^{\alpha}(a, b)\right)} \leq C$ for some $C>0$, then in view of (10), we have (21a) and (21b). Since $\left\|y_{n}\right\|_{L^{2}\left(0, T ; L^{2}(a, b)\right)} \leq C$, it then follows from Lemma 2.1 that $\left\|I_{a^{+}}^{1-\alpha} y_{n}\right\|_{L^{2}\left(0, T ; L^{2}(a, b)\right)} \leq C$, which in addition to the fact that $\mathbb{D}_{a^{+}}^{\alpha} y_{n}=\frac{d}{d t} I_{a^{+}}^{1-\alpha} y_{n}$ and that (21b) holds true implies that $\left\|I_{a^{+}}^{1-\alpha} y_{n}\right\|_{H^{1}\left(0, T ; L^{2}(a, b)\right)} \leq C$.

Lemma 2.9. Under the assumption of Lemma 2.8, there exists $y \in L^{2}\left(0, T ; H_{a^{+}}^{\alpha}(a, b)\right)$ such that

$$
y_{n} \rightarrow \quad y \text { weakly in } L^{2}\left(0, T ; H_{a^{+}}^{\alpha}(a, b)\right),
$$

$I_{a^{+}}^{1-\alpha} y_{n} \rightarrow I_{a^{+}}^{1-\alpha} y$ weakly in $L^{2}\left(0, T ; L^{2}(a, b)\right)$,

$I_{a^{+}}^{1-\alpha} y_{n} \rightarrow I_{a^{+}}^{1-\alpha} y$ weakly in $H^{1}\left(0, T ; L^{2}(a, b)\right)$,

$I_{a^{+}}^{1-\alpha} y_{n} \rightarrow \quad I_{a^{+}}^{1-\alpha} y$ strongly in $\mathcal{C}(0, T ;[a, b])$. 
Proof. If $\left\|y_{n}\right\|_{L^{2}\left(0, T ; H_{a^{+}}^{\alpha}(a, b)\right)} \leq C$, then there exists $y \in L^{2}\left(0, T ; H_{a^{+}}^{\alpha}(a, b)\right)$ such that (22a) holds true. This means that

$$
\begin{aligned}
y_{n} & \rightarrow y \text { weakly in } L^{2}\left(0, T ; L^{2}(a, b)\right), \\
\mathbb{D}_{a^{+}}^{\alpha} y_{n} & \rightarrow \mathbb{D}_{a^{+}}^{\alpha} y \text { weakly in } L^{2}\left(0, T ; L^{2}(a, b)\right) .
\end{aligned}
$$

Now, let $\phi \in L^{2}\left(0, T ; L^{2}(a, b)\right)$. Then in view of Lemma 2.1, we have that $I_{b^{-}}^{1-\alpha} \phi \in L^{2}\left(0, T ; L^{2}(a, b)\right)$ and we can write

$$
\begin{aligned}
\int_{0}^{T} \int_{a}^{b} I_{a^{+}}^{1-\alpha} y_{n} \phi d x d t & =\frac{1}{\Gamma(1-\alpha)} \int_{0}^{T} \int_{a}^{b}\left(\int_{a}^{x}(x-s)^{-\alpha} y_{n}(s) d s\right) \phi(x) d x d t \\
& =\frac{1}{\Gamma(1-\alpha)} \int_{0}^{T} \int_{a}^{b} y_{n}(s)\left(\int_{s}^{b}(x-s)^{-\alpha} \phi(x) d x\right) d s d t \\
& =\int_{0}^{T} \int_{a}^{b} y_{n} I_{b^{-}}^{1-\alpha} \phi d x d t .
\end{aligned}
$$

Passing to the limit in this latter identity while using (23), we obtain that

$$
\begin{aligned}
\int_{0}^{T} \int_{a}^{b} I_{a^{+}}^{1-\alpha} y_{n} \phi d x d t & \rightarrow \int_{0}^{T} \int_{a}^{b} y I_{b^{-}}^{1-\alpha} \phi d x d t \\
& =\frac{1}{\Gamma(1-\alpha)} \int_{0}^{T} \int_{a}^{b} y(s)\left(\int_{s}^{b}(x-s)^{-\alpha} \phi(x) d x\right) d s d t \\
& =\frac{1}{\Gamma(1-\alpha)} \int_{0}^{b_{a}} \int_{a}^{x}\left(\int_{a}^{x}(x-s)^{-\alpha} y(s) d s\right) \phi(x) d x d t \\
& =\int_{0}^{T} \int_{a}^{b} I_{a^{+}}^{1-\alpha} y \phi d x d t
\end{aligned}
$$

from which we deduce (22b). From (21d), we have (22c) and since the imbedding of $H^{1}\left(0, T ; L^{2}(a, b)\right)$ into $\mathcal{C}(0, T ;[a, b])$ is compact, we deduce $(22 \mathrm{~d})$.

\section{Optimal boundary control for a parabolic fractional Sturm-Liouville problem on a single edge}

\subsection{Existence results of boundary value fractional diffusion Sturm-Liouville equations}

We start with some existence results concerning homogeneous and nonhomo- geneous fractional diffusion Sturm-Liouville equations. We look at the following problem:

$$
\left\{\begin{array}{lll}
\partial_{t} y+\mathcal{D}_{b^{-}}^{\alpha} \beta \mathbb{D}_{a^{+}}^{\alpha} y+q y & =f & \text { in }(a, b) \times(0, T) \\
I_{a^{+}}^{1-\alpha} y\left(a^{+}\right) & =0 & \text { in }(0, T) \\
\beta(b) \mathbb{D}_{a^{+}}^{\alpha} y\left(b^{-}\right) & =0 & \text { in }(0, T) \\
y(0) & =y^{0} & \text { in }(a, b)
\end{array}\right.
$$


We define the set $V$ by:

$$
V=\left\{\rho \in \mathcal{V} \text { such that } \beta(b) \mathbb{D}_{a^{+}}^{\alpha} y\left(b^{-}\right)=I_{a^{+}}^{1-\alpha} y\left(a^{+}\right)=0\right\} .
$$

Because $V$ is closed in $\mathcal{V}, V$ endowed with norm on $H_{a^{+}}^{\alpha}(a, b)$ is also a Hilbert space.

Theorem 3.1. The space $H_{a^{+}}^{\alpha}$ is separable with respect to the norm $\|\cdot\|_{H_{a^{+}}^{\alpha}}$ for any $\alpha>0$.

Proof. We consider $H_{a^{+}}^{\alpha}$ with the norm $\|\cdot\|_{H_{a^{+}}^{\alpha}}$ and define a mapping

$$
\begin{aligned}
\lambda: & H_{a^{+}}^{\alpha} \rightarrow L^{2} \times L^{2} \\
& u \mapsto\left(u, \mathbb{D}_{a^{+}}^{\alpha} u\right)
\end{aligned}
$$

$\lambda\left(H_{a^{+}}^{\alpha}\right)$ is separable as a subset of separable space $L^{2} \times L^{2}$. Since $\lambda$ is isometry, $\lambda\left(H_{a^{+}}^{\alpha}\right)=H_{a^{+}}^{\alpha}$ is also separable with respect to the norm $\|\cdot\|_{H_{a^{+}}^{\alpha}}$.

Corollary 3.1. The Hilbert space $V$ is separable as a subset of separable space $H_{a^{+}}^{\alpha}$.

We denote by $\langle.,$.$\rangle the dual product of V^{*}$ and $V$ and $(.,$.$) by the scalar product in L^{2}(a, b)$. We refer to $L^{2}\left(0, T ; L^{2}(a, b)\right)$ as the Hilbert space equipped with the norm

$$
\|y\|_{L^{2}\left(0, T ; L^{2}\right)}^{2}:=\int_{0}^{T}\|y(t)\|_{L^{2}(a, b)}^{2} d t
$$

and defined $L^{2}(0, T ; V)$ and its norm analogously. Moreover, we denote by $W(0, T)$ the linear space of all $y \in L^{2}(0, T ; V)$ having derivative $\partial_{t} y \in L^{2}\left(0, T ; V^{*}\right)$, equipped with the norm

$$
\|y\|_{W(0, T)}^{2}=\int_{0}^{T}\left(\|y(t)\|_{V}^{2}+\left\|\partial_{t} y(t)\right\|_{V^{*}}^{2}\right) d t .
$$

The space $W(0, T)=\left\{y \in L^{2}(0, T ; V): \partial_{t} y \in L^{2}\left(0, T ; V^{*}\right)\right\}$ is a Hilbert space with the scalar product

$$
(y, \phi)_{W(0, T)}=\int_{0}^{T}(y(t), \phi(t))_{V} d t+\int_{0}^{T}\left(\partial_{t} y(t), \partial_{t} \phi(t)\right)_{V^{*}} d t .
$$

Lemma 3.1. The space $W(0, T)$ is continuously emdedded in $C\left([0, T] ; L^{2}(a, b)\right)$.

It follows that for any $y \in W(0, T)$, the values $y(0)$ and $y(T)$ exists and belong to $L^{2}(a, b)$.

Remark 2. The operator $\mathcal{D}_{b^{-}}^{\alpha}\left(\beta \mathbb{D}_{a^{+}}^{\alpha} y\right)$ is a self adjoint and positive on $V$. Indeed, from (18), we have that for any $y \in V$,

$$
\int_{a}^{b} \mathcal{D}_{b^{-}}^{\alpha}\left(\beta \mathbb{D}_{a^{+}}^{\alpha} y\right)(s) y(s) d s=\int_{a}^{b} y(s) \mathcal{D}_{b^{-}}^{\alpha}\left(\beta \mathbb{D}_{a^{+}}^{\alpha} \phi\right)(s) d s
$$

and

$$
\int_{a}^{b} \mathcal{D}_{b^{-}}^{\alpha}\left(\beta \mathbb{D}_{a^{+}}^{\alpha} y\right)(s) y(s) d s=\int_{a}^{b} \beta\left|\mathbb{D}_{a^{+}}^{\alpha}(y)(s)\right|^{2} d s \geq 0 .
$$


The following lemma gives the weak formulation of problem (25).

Lemma 3.2. For $f \in L^{2}\left(0, T ; L^{2}(a, b)\right), y^{0} \in L^{2}(a, b)$ the problem: find $y \in W(0, T)$ such that

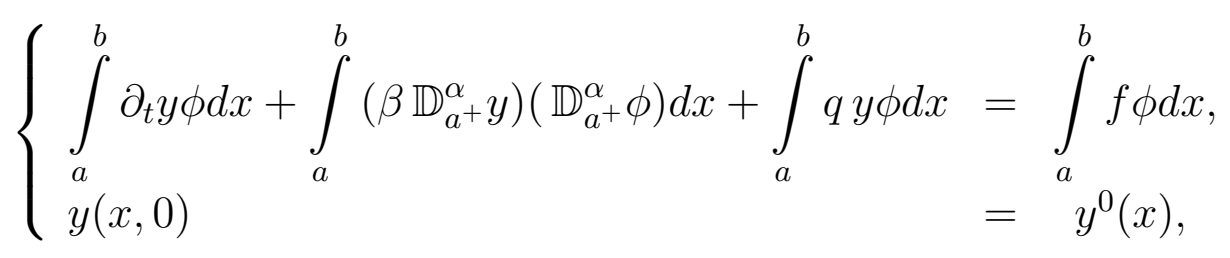

is equivalent to problem (25), and it is called the weak formulation of (25).

Using the fact that

$$
\int_{a}^{b} \partial_{t} y(x, t) \phi(x) d x=\frac{d}{d t} \int_{a}^{b} y(x, t) \phi(x) d x \quad \forall \phi \in V,
$$

problem (25) can be transform as: find $y \in W(0, T)$ such that

$$
\frac{d y}{d t}+\mathcal{A}(t) y=f
$$

and

$$
y(0)=y^{0}
$$

Thus, problem (25) becomes

$$
\left\{\begin{array}{l}
y \in W(0, T) \\
\frac{d}{d t}(y(t), \phi)+a(t ; y(t), \phi)=(f(t), \phi) \forall \phi \in V, \\
y(0)=y^{0} .
\end{array}\right.
$$

Let us give our definition of weak solution to problem (25).

Definition 3.1. We say that a function $y \in L^{2}(0, T ; V)$, such that $\partial_{t} y \in L^{2}\left(0, T ; V^{*}\right)$ is a weak solution for problem (25) if

$$
\int_{0}^{T}\left(\partial_{t} y, \varphi\right) d t+\int_{0}^{T} a(y, \varphi) d t=\int_{0}^{T}(f, \varphi) d t
$$

for all $\varphi \in L^{2}(0, T ; V)$ such that $\partial_{t} \varphi \in L^{2}\left(0, T ; V^{*}\right), \varphi(T)=0$, and $y(0)=y^{0}$ in the sense of $L^{2}(a, b)$.

Theorem 3.2. Let $f \in L^{2}\left(0, T ; L^{2}(a, b)\right)$ and $q \in L^{\infty}(a, b)$ and $\beta \in \mathcal{C}(a, b)$ be such that $\beta>\beta_{0}>0$ and $q \geq q_{0}>0$. Then, the problem (25) has a unique solution $y \in W(0, T)$.

Proof. Uniqueness. Let $y_{1}$ and $y_{2}$ be two solution of equation (31). By setting $y=y_{1}-y_{2}, y$ satisfy (31) with $f=0, y^{0}=0$. Taking the scalar product with $y(t)$ under the duality between $V$ and $V^{*}$, we get

$$
a(t ; y(t), y(t))+\left(\frac{d y(t)}{d t}, y(t)\right)=0
$$


Since

$$
\int_{0}^{T}\left(\frac{d y(t)}{d t}, y(t)\right) d t=\frac{1}{2}\|y(T)\|_{L^{2}(a, b)}^{2}
$$

equation (35) becomes

$$
a(t ; y(t), y(t))+\frac{1}{2}\|y(T)\|_{L^{2}(a, b)}^{2}=0
$$

Using the coercivity of the bilinear functional, we have

$$
\min \left(\beta_{0}, q_{0}\right) \int_{0}^{T}\|y(t)\|_{V}^{2} d t+\frac{1}{2}\|y(T)\|_{L^{2}(a, b)}^{2} \leq 0
$$

and thus

$$
y=0 \text {. }
$$

Existence. The space $V$ is a separable Hilbert space (see corollary 3.1). Therefore there exists a countable set which is dense in $V$. We may then find a basis $w_{1}, w_{2}, \cdots, w_{m}, \cdots$ in $V$ in the following sense:

$$
\left\{\begin{array}{l}
w_{i} \in V \text { for all } i \\
\forall m, w_{1}, w_{2}, \cdots, w_{m} \text { are linearly independent; } \\
\text { the linear combination } \sum_{i=1}^{m} \phi_{j} w_{j}, \phi_{j} \in \mathbb{R} \text { are dense in } V .
\end{array}\right.
$$

We then find $y_{m}=y_{m}(t)$ "approximate solution of the problem (31), (32) in the form

$$
y_{m}(t)=\sum_{i=1}^{m} g_{i m}(t) w_{i}
$$

where the $g_{i m}$ are chosen such that

$$
\left(\frac{d y(t)}{d t}, w_{j}\right)+a\left(t ; y_{m}(t), w_{j}\right)=\left(f(t), w_{j}\right), \quad 1 \leq j \leq m
$$

and

$$
y_{m}(0)=y_{0 m}=\sum_{i=1}^{m} \varepsilon_{i m} w_{i}, \quad \sum_{i=1}^{m} \varepsilon_{i m} w_{i} \rightarrow y^{0} \text { in } L^{2}(a, b) \text { as } m \rightarrow \infty,
$$

where

$$
a\left(t ; y(t), w_{j}\right)=\int_{a}^{b} \beta(x) \mathbb{D}_{a^{+}}^{\alpha} y \mathbb{D}_{a^{+}}^{\alpha} w_{j} d x+\int_{a}^{b} q y w_{j} d x
$$

System (40), (41) is a system of $m$ linear differential equations in $g_{i m}(t)$ of the form

$$
\mathcal{W}_{m} \frac{d g_{m}}{d t}+\mathcal{A}_{m}(t) g_{m}=f_{m}, \quad g_{m}(0)=\left\{\varepsilon_{i m}\right\}
$$


where

$$
\begin{array}{ll}
\mathcal{W}_{m}=\left\|\left(w_{i}, w_{j}\right)\right\| ; & \mathcal{A}_{m}(t)=\left\|a\left(t ; w_{i}, w_{j}\right)\right\|, \\
g_{m}(t)=\left\{g_{i m}(t)\right\}, & f_{m}(t)=\left\{\left(f(t), w_{j}\right)\right\} .
\end{array}
$$

Since $\operatorname{det} \mathcal{W}_{m} \neq 0$, problem (40), (41) admits a unique solution. It remain to show that as $m \rightarrow \infty$, $y_{m} \rightarrow y, y$ being a solution of (31), (32).

Multiplying equation (40) by $g_{j m}(t)$ and summing over $j$, we obtain

$$
\left(\frac{d y_{m}(t)}{d t}, y_{m}(t)\right)+a\left(t ; y_{m}(t), y_{m}(t)\right)=\left(f(t), y_{m}(t)\right)
$$

that is

$$
\frac{1}{2} \frac{d}{d t}\left\|y_{m}(t)\right\|_{L^{2}(a, b)}^{2}+a\left(t ; y_{m}(t), y_{m}(t)\right)=\left(f(t), y_{m}(t)\right)
$$

Using the coercivity of $a$, Cauchy Schwarz and Young inequalities, we get

$$
\begin{aligned}
& \left\|y_{m}(T)\right\|_{L^{2}(a, b)}^{2}+2 \min \left(\beta_{0}, q_{0}\right) \int_{0}^{T}\left\|y_{m}(t)\right\|_{V}^{2} d t \\
& \leq\left\|y_{0 m}\right\|_{L^{2}(a, b)}^{2}+2 \int_{0}^{T}\left|\left(f(t), y_{m}(t)\right)\right| d t \\
& \leq\left\|y_{0 m}\right\|_{L^{2}(a, b)}^{2}+2 \int_{0}^{T} \|\left(f(t)\left\|_{V^{*}}\right\| y_{m}(t) \|_{V} d t\right. \\
& \leq\left\|y_{0 m}\right\|_{L^{2}(a, b)}^{2}+\frac{1}{\min \left(\beta_{0}, q_{0}\right)} \int_{0}^{T} \|\left(f(t)\left\|_{V^{*}}^{2} d t+\int_{0}^{T}\right\| y_{m}(t) \|_{V}^{2} d t\right.
\end{aligned}
$$

From (41) we have $\left\|y_{0 m}\right\|_{L^{2}(a, b)} \geq C\left\|y^{0}\right\|_{L^{2}(a, b)}$ and therefore

$$
\int_{0}^{T}\left\|y_{m}(t)\right\|_{V^{2}}^{2} d t \leq C\left(\left\|y^{0}\right\|_{L^{2}(a, b)}^{2}+\int_{0}^{T}\|f(t)\|_{V^{*}}^{2} d t\right) .
$$

Equation (45) implies that the sequence $y_{m}$ is bounded in $L^{2}(0, T ; V)$ and we may extract a subsequence $y_{\mu}$ such that

$$
y_{\mu} \rightarrow z \text { weakly in } L^{2}(0, T ; V) .
$$

Let $j$ be fixed but arbitrary and let $\mu>j$. Then (40) is valid with $m=\mu$.

Multiply both sides of (40) by

$$
\phi \in V, \quad \phi(t) \in C^{1}[0, T], \quad \phi(T)=0,
$$

and integrate over $(0, T)$ while setting $\phi_{j}=\phi(t) w_{j}$, we have

$$
\int_{0}^{T}\left[-\left(y_{\mu}(t), \phi_{j}^{\prime}\right)+a\left(t ; y_{\mu}(t), \phi_{j}\right)\right] d t=\int_{0}^{T}\left(f, \phi_{j}\right) d t+\left(y_{0 \mu}, \phi_{j}(0)\right) \text {. }
$$


Using (46), we can pass to the limit in (48). We then obtain

$$
\int_{0}^{T}\left[-\left(z, \phi_{j}^{\prime}\right)+a\left(t ; z, \phi_{j}\right)\right] d t=\int_{0}^{T}\left(f, \phi_{j}\right) d t+\left(y^{0}, \phi_{j}(0)\right) .
$$

Equation (49) is true for any $\phi$ satisfying (47). Therefore we may take $\phi \in C_{c}(0, T)$ and hence (49) gives

$$
\frac{d}{d t}\left(z(t), w_{j}\right)+a\left(t ; z(t), w_{j}\right)=\left(f(t), w_{j}\right)
$$

But in (50) $j$ is arbitrary and since finite linear combination of $w_{j}$ are dense in $V$,we deduce

$$
\frac{d z}{d t}+\mathcal{A}(t) z=f
$$

Therefore,

$$
\frac{d z}{d t}=f-\mathcal{A}(t) z \in L^{2}\left(0, T ; V^{*}\right) \quad \text { and hence } z \in W(0, T) .
$$

This allow us to integrate by part in $t$. Thus, taking into account (51) we obtain

$$
\left(z(0), w_{j}\right) \phi(0)=\left(y^{0}, w_{j}\right) \phi(0) \quad \forall j, \forall \phi .
$$

That is $\left(z(0), w_{j}\right) \phi(0)=\left(y^{0}, w_{j}\right) \phi(0) \forall j$ and thus $z(0)=y^{0}$. Hence $z$ is solution and therefore $z=y$ is a solution. We may then replace (46) by

$$
y_{m} \rightarrow y \text { weakly in } L^{2}(0, T ; V)
$$

The estimate (45) give us

$$
\|y\|_{L^{2}(0, T ; V)}^{2} \leq C\left(\left\|y^{0}\right\|_{L^{2}(a, b)}^{2}+\int_{0}^{T}\|f(t)\|_{V^{*}}^{2} d t\right) .
$$

Moreover, since $\frac{d z}{d t}=f-\mathcal{A}(t) z$, we have using (55)

$$
\left\|\frac{d y}{d t}\right\|_{L^{2}\left(0, T ; V^{*}\right)}^{2} \leq C^{\prime}\left(\left\|y^{0}\right\|_{L^{2}(a, b)}^{2}+\int_{0}^{T}\|f(t)\|_{V^{*}}^{2} d t\right) .
$$

Corollary 3.2. Let $f \in L^{2}\left(0, T ; L^{2}(a ; b)\right), q \in L^{\infty}(a, b)$, and $\beta \in C(a, b)$, then there exist a unique $y \in W(0, T)$ solution of the following problem

$$
\left\{\begin{array}{lll}
\partial_{t} y+\mathcal{D}_{b^{-}}^{\alpha}\left(\beta \mathbb{D}_{a^{+}}^{\alpha} y\right)+q y & =f \quad \text { in }(a, b) \times(0, T) \\
I_{a^{+}}^{1-\alpha} y\left(a^{+}\right) & =0 & \text { in }(0, T) \\
\beta(b) \mathbb{D}_{a^{+}}^{\alpha} y\left(b^{-}\right) & =0 & \text { in }(0, T) \\
y(0) & = & 0
\end{array}\right.
$$

Proof. The proof comes immediatly from theorem 3.2. 
Lemma 3.3. Let $h \in L^{2}\left(0, T ; L^{2}(a ; b)\right), q \in L^{\infty}(a, b)$, and $\beta \in C(a, b)$, then there exist a unique $\phi \in W(0, T)$ solution of the following problem

$$
\left\{\begin{array}{llll}
-\partial_{t} \phi+\mathcal{D}_{b^{-}}^{\alpha}\left(\beta \mathbb{D}_{a^{+}}^{\alpha} \phi\right)+q \phi & = & \text { in }(a, b) \times(0, T), \\
I_{a^{+}}^{1-\alpha} \phi\left(a^{+}\right) & =0 & \text { in }(0, T), \\
\beta(b) \mathbb{D}_{a^{+}}^{\alpha} \phi\left(b^{-}\right) & =0 & \text { in }(0, T), \\
\phi(T) & = & 0 & \text { in }(a, b) .
\end{array}\right.
$$

Proof. By considering the change of variable $t=T-t$ into (57), we have the result.

Now consider the following inhomogeneous boundary value problem:

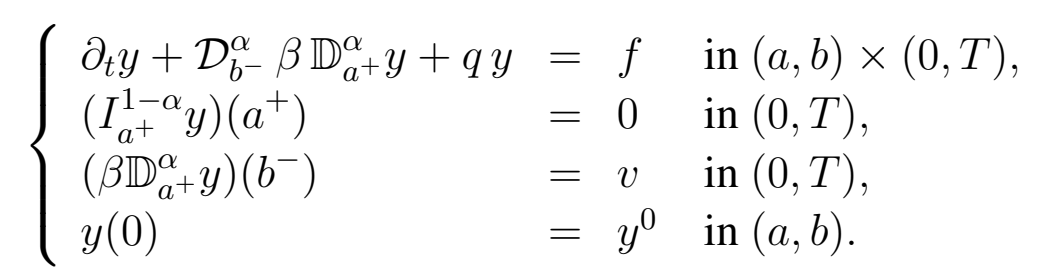

We define the set $V_{0}$ by:

$$
V_{0}=\left\{y \in \mathcal{V} \text { such that } I_{a^{+}}^{1-\alpha} y\left(a^{+}\right)=0\right\} .
$$

Then $V_{0}$ is closed in $\mathcal{V}$. Hence $V_{0}$ endowed with norm on $H_{a^{+}}^{\alpha}(a, b)$ defined by (10) is also a Hilbert space.

Corollary 3.3. The Hilbert space $V_{0}$ is separable as a subset of separable space $H_{a^{+}}^{\alpha}$.

We denote by $W_{0}(0, T)$ the linear space of all $y \in L^{2}\left(0, T ; V_{0}\right)$ having derivative $\partial_{t} y \in L^{2}\left(0, T ; V_{0}^{*}\right)$, equipped with the norm

$$
\|y\|_{W_{0}(0, T)}^{2}=\int_{0}^{T}\left(\|y(t)\|_{V_{0}}^{2}+\left\|\partial_{t} y(t)\right\|_{V_{0}^{*}}^{2}\right) d t .
$$

The space $W_{0}(0, T)=\left\{y \in L^{2}\left(0, T ; V_{0}\right): \partial_{t} y \in L^{2}\left(0, T ; V_{0}^{*}\right)\right\}$ is a Hilbert space with the scalar product

$$
(y, \phi)_{W_{0}(0, T)}=\int_{0}^{T}(y(t), \phi(t))_{V_{0}} d t+\int_{0}^{T}\left(\partial_{t} y(t), \partial_{t} \phi(t)\right)_{V_{0}^{*}} d t .
$$

Lemma 3.4. The space $W_{0}(0, T)$ is continuously emdedded in $C\left([0, T] ; L^{2}(a, b)\right)$.

It follows that for any $y \in W_{0}(0, T)$, the values $y(0)$ and $y(T)$ exists and belong to $L^{2}(a, b)$.

If we multiply the first equation in (59) by $\phi \in V_{0}$, then integrate by parts on over $(a, b)$ while using (18), we obtain that

$$
\int_{a}^{b} \partial_{t} y \phi d x+\int_{a}^{b}\left(\beta \mathbb{D}_{a^{+}}^{\alpha} y\right)\left(\mathbb{D}_{a^{+}}^{\alpha} \phi\right) d x+\int_{a}^{b} q y \phi d x=\int_{a}^{b} f \phi d x+v\left(I_{a^{+}}^{1-\alpha} \phi\right)\left(b^{-}\right) .
$$

The following lemma gives the weak formulation of problem (59). 
Lemma 3.5. For $f \in L^{2}\left(0, T ; L^{2}(a, b)\right), y^{0} \in L^{2}(a, b)$ the problem: find $y \in W_{0}(0, T)$ such that

$$
\left\{\begin{aligned}
\int_{a}^{b} \partial_{t} y \phi d x+\int_{a}^{b}\left(\beta \mathbb{D}_{a^{+}}^{\alpha} y\right)\left(\mathbb{D}_{a^{+}}^{\alpha} \phi\right) d x+\int_{a}^{b} q y \phi d x & =\int_{a}^{b} f \phi d x+v\left(I_{a^{+}}^{1-\alpha} \phi\right)\left(b^{-}\right), \\
y(x, 0) & =y^{0}(x),
\end{aligned}\right.
$$

is equivalent to problem (59), and it is called the weak formulation of (59).

\section{Since}

$$
\int_{a}^{b} \partial_{t} y(x, t) \phi(x) d x=\frac{d}{d t} \int_{a}^{b} y(x, t) \phi(x) d x \quad \forall \phi \in V_{0},
$$

problem (59) can be transform as: find $y \in W_{0}(0, T)$ such that

$$
\frac{d y}{d t}+\mathcal{A}(t) y=f+v\left(I_{a^{+}}^{1-\alpha} \phi\right)\left(b^{-}\right)
$$

and

$$
y(0)=y^{0}
$$

Thus, problem (59) becomes

$$
\left\{\begin{array}{l}
y \in W_{0}(0, T), \\
\frac{d}{d t}(y(t), \phi)+a(t ; y(t), \phi)=(f(t), \phi)+v\left(I_{a^{+}}^{1-\alpha} \phi\right)\left(b^{-}\right) \forall \phi \in V_{0}, \\
y(0)=y^{0} .
\end{array}\right.
$$

Theorem 3.3. Let $f \in L^{2}\left(0, T ; L^{2}(a, b)\right)$ and $q \in L^{\infty}(a, b)$ and $\beta \in \mathcal{C}(a, b)$ be such that $\beta>\beta_{0}>0$ and $q \geq q_{0}>0$. Then, the problem has a unique solution $y \in W_{0}(0, T)$.

Proof. Uniqueness. Let $y_{1}$ and $y_{2}$ be two solution of equation (65). By setting $y=y_{1}-y_{2}, y$ satisfy (65) with $f=0, y^{0}=0, v=0$. Taking the scalar product with $y(t)$ under the duality between $V$ and $V^{*}$, we get

$$
a(t ; y(t), y(t))+\left(\frac{d y(t)}{d t}, y(t)\right)=0
$$

Since

$$
\int_{0}^{T}\left(\frac{d y(t)}{d t}, y(t)\right) d t=\frac{1}{2}\|y(T)\|_{L^{2}(a, b)}^{2},
$$

equation (68) becomes

$$
a(t ; y(t), y(t))+\frac{1}{2}\|y(T)\|_{L^{2}(a, b)}^{2}=0
$$

Using the coercivity of the bilinear functional, we have

$$
\min \left(\beta_{0}, q_{0}\right) \int_{0}^{T}\|y(t)\|_{V}^{2} d t+\frac{1}{2}\|y(T)\|_{L^{2}(a, b)}^{2} \leq 0
$$


and thus

$$
y=0 .
$$

Existence. The space $V_{0}$ is a separable Hilbert space (see corollary 3.3). Therefore there exists a countable set which is dense in $V_{0}$. We may then find a basis $w_{1}, w_{2}, \cdots, w_{m}, \cdots$ in $V_{0}$ in the following sense:

$$
\left\{\begin{array}{l}
w_{i} \in V_{0} \text { for all } i \\
\forall m, w_{1}, w_{2}, \cdots, w_{m} \text { are linearly independent; } \\
\text { the linear combination } \sum_{i=1}^{m} \phi_{j} w_{j}, \phi_{j} \in \mathbb{R} \text { are dense in } V_{0} .
\end{array}\right.
$$

We then find $y_{m}=y_{m}(t)$ "approximate solution of the problem (65), (66) in the form

$$
y_{m}(t)=\sum_{i=1}^{m} g_{i m}(t) w_{i}
$$

where the $g_{i m}$ are chosen such that

$$
\left(\frac{d y(t)}{d t}, w_{j}\right)+a\left(t ; y_{m}(t), w_{j}\right)=\left(f(t), w_{j}\right)+v\left(I_{a^{+}}^{1-\alpha} w_{j}\right)\left(b^{-}\right), \quad 1 \leq j \leq m,
$$

and

$$
y_{m}(0)=y_{0 m}=\sum_{i=1}^{m} \varepsilon_{i m} w_{i}, \quad \sum_{i=1}^{m} \varepsilon_{i m} w_{i} \rightarrow y^{0} \text { in } L^{2}(a, b) \text { as } m \rightarrow \infty,
$$

where

$$
a\left(t ; y(t), w_{j}\right)=\int_{a}^{b} \beta(x) \mathbb{D}_{a^{+}}^{\alpha} y \mathbb{D}_{a^{+}}^{\alpha} w_{j} d x+\int_{a}^{b} q y w_{j} d x
$$

System (73), (74) is a system of $m$ linear differential equations in $g_{i m}(t)$ of the form

$$
\mathcal{W}_{m} \frac{d g_{m}}{d t}+\mathcal{A}_{m}(t) g_{m}=f_{m}, \quad g_{m}(0)=\left\{\varepsilon_{i m}\right\}
$$

where

$$
\begin{array}{ll}
\mathcal{W}_{m}=\left\|\left(w_{i}, w_{j}\right)\right\| ; & \mathcal{A}_{m}(t)=\left\|a\left(t ; w_{i}, w_{j}\right)\right\|, \\
g_{m}(t)=\left\{g_{i m}(t)\right\}, \quad f_{m}(t)=\left\{\left(f(t), w_{j}\right)+v\left(I_{a^{+}}^{1-\alpha} w_{j}\right)\left(b^{-}\right)\right\} .
\end{array}
$$

Since $\operatorname{det} \mathcal{W}_{m} \neq 0$, problem (73), (74) admits a unique solution. It remain to show that as $m \rightarrow \infty$, $y_{m} \rightarrow y, y$ being a solution of (65), (66).

Multiplying equation (73) by $g_{j m}(t)$ and summing over $j$, we obtain

$$
\left(\frac{d y_{m}(t)}{d t}, y_{m}(t)\right)+a\left(t ; y_{m}(t), y_{m}(t)\right)=\left(f(t), y_{m}(t)\right)+v\left(I_{a^{+}}^{1-\alpha} y_{m}\right)\left(b^{-}\right)
$$

that is

$$
\frac{1}{2} \frac{d}{d t}\left\|y_{m}(t)\right\|_{L^{2}(a, b)}^{2}+a\left(t ; y_{m}(t), y_{m}(t)\right)=\left(f(t), y_{m}(t)\right)+v\left(I_{a^{+}}^{1-\alpha} y_{m}\right)\left(b^{-}\right) .
$$


Observing that for any $y_{m} \in V_{0}$,

$$
\begin{aligned}
& \left|I_{a^{+}}^{1-\alpha} y_{m}\left(b^{-}\right)\right|=\left|I_{a^{+}}^{1-\alpha} y_{m}\left(b^{-}\right)-I_{a^{+}}^{1-\alpha} y_{m}\left(a^{+}\right)\right| \\
& =\left|\int_{a}^{b} \frac{d}{d x}\left(I_{a^{+}}^{1-\alpha} y_{m}\right)(x) d x\right| \\
& =\left|\int_{a}^{b} \mathbb{D}_{a^{+}}^{\alpha} y_{m}(x) d x\right| \\
& \leq|b-a|^{1 / 2}\left(\int_{a}^{b}\left(\mathbb{D}_{a^{+}}^{\alpha} y_{m}(x)\right)^{2} d x\right)^{1 / 2} \\
& \leq|b-a|^{1 / 2}\left\|\mathbb{D}_{a^{+}}^{\alpha} y_{m}\right\|_{L^{2}(a, b)},
\end{aligned}
$$

and using the coercivity of $a$, Cauchy Schwarz and Young inequalities, we get

$$
\begin{aligned}
& \left\|y_{m}(T)\right\|_{L^{2}(a, b)}^{2}+2 \min \left(\beta_{0}, q_{0}\right) \int_{0}^{T}\left\|y_{m}(t)\right\|_{V_{0}}^{2} d t+2 \int_{0}^{T} v\left(I_{a^{+}}^{1-\alpha} y_{m}\right)\left(b^{-}\right) d t \\
& \leq\left\|y_{0 m}\right\|_{L^{2}(a, b)}^{2}+2 \int_{0}^{T}\left|\left(f(t), y_{m}(t)\right)\right| d t+2|b-a|^{1 / 2} \int_{0}^{T} v\left\|_{a^{+}}^{\alpha} y_{m}\right\|_{L^{2}(a, b)} d t \\
& \leq\left\|y_{0 m}\right\|_{L^{2}(a, b)}^{2}+2 \int_{0}^{T}\|f(t)\|_{V_{0}^{*}}\left\|y_{m}(t)\right\|_{V_{0}} d t+|b-a|^{1 / 2} \int_{0}^{T} v^{2} d t \\
& +|b-a|^{1 / 2} \int_{0}^{T}\left\|\mathbb{D}_{a^{+}}^{\alpha} y_{m}\right\|_{L^{2}(a, b)}^{2} d t \\
& \leq\left\|y_{0 m}\right\|_{L^{2}(a, b)}^{2}+\frac{1}{\min \left(\beta_{0}, q_{0}\right)} \int_{0}^{T}\|f(t)\|_{V_{0}^{*}}^{2} d t+\int_{0}^{T}\left\|y_{m}(t)\right\|_{V_{0}}^{2} d t \\
& +|b-a|^{1 / 2} \int_{0}^{T} v^{2} d t+|b-a|^{1 / 2} \int_{0}^{T}\left\|\mathbb{D}_{a^{+}}^{\alpha} y_{m}\right\|_{L^{2}(a, b)}^{2} d t
\end{aligned}
$$

From (74) we have $\left\|y_{0 m}\right\|_{L^{2}(a, b)} \geq C\left\|y^{0}\right\|_{L^{2}(a, b)}$ and therefore

$$
\int_{0}^{T}\left\|y_{m}(t)\right\|_{V_{0}}^{2} d t \leq C\left(\left\|y^{0}\right\|_{L^{2}(a, b)}^{2}+\int_{0}^{T}\|f(t)\|_{V_{0}^{*}}^{2} d t+\int_{0}^{T} v^{2} d t\right) .
$$

Equation (78) implies that the sequence $y_{m}$ is bounded in $L^{2}\left(0, T ; V_{0}\right)$ and we may extract a subsequence $y_{\mu}$ such that

$$
y_{\mu} \rightarrow z \text { weakly in } L^{2}\left(0, T ; V_{0}\right)
$$

Let $j$ be fixed but arbitrary and let $\mu>j$. Then (73) is valid with $m=\mu$. 
Multiply both sides of (73) by

$$
\phi \in V_{0}, \quad \phi(t) \in C^{1}[0, T], \quad \phi(T)=0
$$

and integrate over $(0, T)$ while setting $\phi_{j}=\phi(t) w_{j}$, we have

$$
\int_{0}^{T}\left[-\left(y_{\mu}, \phi_{j}^{\prime}\right)+a\left(t ; y_{\mu}, \phi_{j}\right)\right] d t=\int_{0}^{T}\left[\left(f, \phi_{j}\right)+v\left(I_{a^{+}}^{1-\alpha} \phi_{j}\right)\left(b^{-}\right)\right] d t+\left(y_{0 \mu}, \phi_{j}(0)\right) .
$$

Using (79), we can pass to the limit in (81). We then obtain

$$
\int_{0}^{T}\left[-\left(z, \phi_{j}^{\prime}\right)+a\left(t ; z, \phi_{j}\right)\right] d t=\int_{0}^{T}\left[\left(f, \phi_{j}\right)+v\left(I_{a^{+}}^{1-\alpha} \phi_{j}\right)\left(b^{-}\right)\right] d t+\left(y^{0}, \phi_{j}(0)\right) .
$$

Equation (82) is true for any $\phi$ satisfying (80). Therefore we may take $\phi \in C_{c}(0, T)$ and hence (82) gives

$$
\frac{d}{d t}\left(z(t), w_{j}\right)+a\left(t ; z(t), w_{j}\right)=\left(f(t), w_{j}\right)+v\left(I_{a^{+}}^{1-\alpha} w_{j}\right)\left(b^{-}\right)
$$

But in (83) $j$ is arbitrary and since finite linear combination of $w_{j}$ are dense in $V_{0}$, we deduce

$$
\frac{d z}{d t}+\mathcal{A}(t) z=f+v\left(I_{a^{+}}^{1-\alpha} w_{j}\right)\left(b^{-}\right)
$$

Therefore,

$$
\frac{d z}{d t}=f+v\left(I_{a^{+}}^{1-\alpha} \phi_{j}\right)\left(b^{-}\right)-\mathcal{A}(t) z \in L^{2}\left(0, T ; V_{0}^{*}\right) \quad \text { and hence } z \in W_{0}(0, T) .
$$

This allow us to integrate by part in $t$. Thus, taking into account (84) we obtain

$$
\left(z(0), w_{j}\right) \phi(0)=\left(y^{0}, w_{j}\right) \phi(0) \quad \forall j, \forall \phi .
$$

That is $\left(z(0), w_{j}\right) \phi(0)=\left(y^{0}, w_{j}\right) \phi(0) \forall j$ and thus $z(0)=y^{0}$. Hence $z$ is solution and therefore $z=y$ is a solution. We may then replace (79) by

$$
y_{m} \rightarrow y \text { weakly in } L^{2}\left(0, T ; V_{0}\right) \text {. }
$$

The estimate (78) give us

$$
\|y\|_{L^{2}\left(0, T ; V_{0}\right)}^{2} \leq C\left(\left\|y^{0}\right\|_{L^{2}(a, b)}^{2}+\int_{0}^{T}\|f(t)\|_{V_{0}^{*}}^{2} d t+\int_{0}^{T} v^{2} d t\right) .
$$

Moreover, since $\frac{d z}{d t}=f+v\left(I_{a^{+}}^{1-\alpha} \phi_{j}\right)\left(b^{-}\right)-\mathcal{A}(t) z$, we have using (88)

$$
\left\|\frac{d y}{d t}\right\|_{L^{2}\left(0, T ; V_{0}^{*}\right)}^{2} \leq C^{\prime}\left(\left\|y^{0}\right\|_{L^{2}(a, b)}^{2}+\int_{0}^{T}\|f(t)\|_{V_{0}^{*}}^{2} d t+\int_{0}^{T} v^{2} d t\right) .
$$

From now on, we use $C(X)$ to denote a positive constant whose value varies from a line to another but depends on $X$. 


\subsection{Optimal boundary control of fractional diffusion Sturm-Liouville equations}

We are concerned with the optimal control problem:

$$
\min _{v \in \mathcal{U}_{a d}} J(y, v),
$$

where

$$
J(v)=\frac{1}{2} \int_{0}^{T} \int_{a}^{b}\left(y(v)-y_{d}\right)^{2} d x d t+\frac{\gamma}{2} \int_{0}^{T} v^{2} d t,
$$

$y=y(v) \in L^{2}\left(0, T ; V_{0}\right)$ satisfies (59), $y_{d} \in L^{2}\left(0, T ; L^{2}(a, b)\right), \gamma>0$ and $\mathcal{U}_{a d}$ is closed convex subset of $\mathbb{R}_{+} \times \mathbb{R}$.

Theorem 3.4. There exists a unique solution $u \in \mathcal{U}_{a d}$ of the optimal control problem (90).

Proof. We have $J(y(v), v) \geq 0$ for all $v \in \mathcal{U}_{a d}$. So, let $\left\{v_{n}\right\} \subset \mathcal{U}_{a d}$ be a minimizing sequence such that

$$
\lim _{n \rightarrow+\infty} J\left(y\left(v_{n}\right), v_{n}\right)=\min _{v \in \mathcal{U}_{a d}} J(y(v), v) .
$$

Then there exists $C>0$ independent of $n$ such that

$$
\left\|v_{n}\right\|_{L^{2}(0, T)} \leq C
$$

Observing that the control $v_{n}$ is associated to the state $y_{n} \in V_{0}$ solution of

$$
\left\{\begin{array}{lll}
\partial_{t} y_{n}+\mathcal{D}_{b^{-}}^{\alpha} \beta \mathbb{D}_{a^{+}}^{\alpha} y_{n}+q y_{n} & =f & \text { in }(a, b) \times(0, T), \\
I_{a^{+}}^{1-\alpha} y_{n}\left(a^{+}\right) & =0 & \text { in }(0, T), \\
\beta(b) \mathbb{D}_{a^{+}}^{\alpha} y_{n}\left(b^{-}\right) & =v_{n} & \text { in }(0, T), \\
y_{n}(0) & =y^{0} & \text { in }(a, b),
\end{array}\right.
$$

it follows from (88) that there exists $C=C\left(\beta_{0},\|f\|_{L^{2}(a, b)},\|q\|_{\infty}\right)>0$, such that

$$
\left\|y_{n}\right\|_{L^{2}\left(0, T ; H_{a^{+}}^{\alpha}(a, b)\right)} \leq C
$$

From (92), (94),the fact that $\mathcal{U}_{a d}$ is a closed convex subset of $\mathbb{R} \times \mathbb{R}_{+}$and Lemma 2.9, we have that there exists $u \in \mathcal{U}_{a d}$ and $y \in L^{2}\left(0, T ; H_{a^{+}}^{\alpha}(a, b)\right)$ such that

$$
\begin{array}{rr}
v_{n} \rightarrow & u \text { in } \mathcal{U}_{a d}, \\
y_{n} & \rightarrow \quad y \text { weakly in } L^{2}\left(0, T ; H_{a^{+}}^{\alpha}(a, b)\right), \\
y_{n} & \rightarrow \quad y \text { weakly in } L^{2}\left(0, T ; L^{2}(a, b)\right), \\
\mathbb{D}_{a^{+}}^{\alpha} y_{n} & \rightarrow \mathbb{D}_{a^{+}}^{\alpha} y \text { weakly in } L^{2}\left(0, T ; L^{2}(a, b)\right), \\
I_{a^{+}}^{1-\alpha} y_{n} & \rightarrow I_{a^{+}}^{1-\alpha} y \text { weakly in } L^{2}\left(0, T ; L^{2}(a, b)\right), \\
I_{a^{+}}^{1-\alpha} y_{n} & \rightarrow I_{a^{+}}^{1-\alpha} y \text { weakly in } H^{1}\left(0, T ; L^{2}(a, b)\right), \\
I_{a^{+}}^{1-\alpha} y_{n} \rightarrow & I_{a^{+}}^{1-\alpha} y \text { strongly in } \mathcal{C}(0, T ;[a, b]) .
\end{array}
$$


From $(93)_{1}$,

$$
\begin{aligned}
\left\|\mathcal{D}_{b^{-}}^{\alpha} \beta \mathbb{D}_{a^{+}}^{\alpha} y_{n}\right\|_{L^{2}\left(0, T ; L^{2}(a, b)\right)} & \leq\|q\|_{\infty}\left\|y_{n}\right\|_{L^{2}\left(0, T ; L^{2}(a, b)\right)}+\|f\|_{L^{2}\left(0, T ; L^{2}(a, b)\right)} \\
& +\left\|\partial_{t} y\right\|_{L^{2}\left(0, T ; L^{2}(a, b)\right)} \\
& \leq C\left(\beta_{0}\|f\|_{L^{2}\left(0, T ; L^{2}(a, b)\right)},\|q\|_{\infty},\left\|y^{0}\right\|_{L^{2}(a, b)}\right) \\
& +\|f\|_{L^{2}\left(0, T ; L^{2}(a, b)\right)} \\
& \leq C\left(\beta_{0},\|f\|_{L^{2}(a, b)},\|q\|_{\infty},\left\|y^{0}\right\|_{L^{2}(a, b)}\right)
\end{aligned}
$$

because of (94) and (21a). Hence there exists $\theta \in L^{2}\left(0, T ; L^{2}(a, b)\right)$ such that

$$
\mathcal{D}_{b^{-}}^{\alpha}\left(\beta \mathbb{D}_{a^{+}}^{\alpha} y_{n}\right) \rightarrow \theta \text { weakly in } L^{2}\left(0, T ; L^{2}(a, b)\right) .
$$

Actually $\theta=\mathcal{D}_{b^{-}}^{\alpha}\left(\beta \mathbb{D}_{a^{+}}^{\alpha} y\right)$. Indeed, let $\phi \in \mathcal{C}_{c}^{1}(0, T ;(a, b))$. Then $\phi, \phi^{\prime} \in L^{2}\left(0, T ; L^{2}(a, b)\right)$ and it follows from Lemma 2.1 that

$$
I_{a^{+}}^{1-\alpha} \phi \in L^{2}\left(0, T ; L^{2}(a, b)\right) \text { and } \mathcal{D}_{a^{+}}^{\alpha} \phi=I_{a^{+}}^{1-\alpha} \phi^{\prime} \in L^{2}\left(0, T ; L^{2}(a, b)\right) .
$$

Hence, $\frac{d}{d t} I_{a^{+}}^{1-\alpha} \phi=\mathbb{D}_{a^{+}}^{\alpha} \phi \in L^{2}\left(0, T ; L^{2}(a, b)\right)$ because $\phi \in \mathcal{C}_{c}^{1}(0, T ;(a, b))$ and

$$
\mathbb{D}_{a^{+}}^{\alpha} \phi=\frac{(t-a)^{-\alpha}}{\Gamma(1-\alpha)} \phi(a)+\mathcal{D}_{a^{+}}^{\alpha} \phi(t)=\mathcal{D}_{a^{+}}^{\alpha} \phi
$$

Consequently, $I_{a^{+}}^{1-\alpha} \phi \in H^{1}\left(0, T ; L^{2}(a, b)\right)$ which is compactly imbedded in $\mathcal{C}((0, T) ;[a, b])$.

So, if we take $\phi \in \mathcal{C}_{c}^{1}(0, T ;(a, b))$ such that $I_{a^{+}}^{1-\alpha} \phi\left(a^{+}\right)=I_{a^{+}}^{1-\alpha} \phi\left(b^{-}\right)=0$ and use (18), we have that

$$
\int_{0}^{T} \int_{a}^{b} \mathcal{D}_{b^{-}}^{\alpha}\left(\beta \mathbb{D}_{a^{+}}^{\alpha} y_{n}\right) \phi d x d t=\int_{0}^{T} \int_{a}^{b}\left(\beta \mathbb{D}_{a^{+}}^{\alpha} y_{n}\right)\left(\mathbb{D}_{a^{+}}^{\alpha} \phi\right) d x d t .
$$

Passing to the limit in this latter identity while using (95d), we obtain that

$$
\int_{0}^{T} \int_{a}^{b} \mathcal{D}_{b^{-}}^{\alpha}\left(\beta \mathbb{D}_{a^{+}}^{\alpha} y_{n}\right) \phi d x d t \rightarrow \int_{0}^{T} \int_{a}^{b}\left(\beta \mathbb{D}_{a^{+}}^{\alpha} y\right)\left(\mathbb{D}_{a^{+}}^{\alpha} \phi\right) d x d t
$$

which after an integration by parts gives

$$
\int_{0}^{T} \int_{a}^{b} \mathcal{D}_{b^{-}}^{\alpha}\left(\beta \mathbb{D}_{a^{+}}^{\alpha} y_{n}\right) \phi d x d t \rightarrow \int_{0}^{T} \int_{a}^{b} \mathcal{D}_{b^{-}}^{\alpha}\left(\beta \mathbb{D}_{a^{+}}^{\alpha} y\right) \phi d x d t
$$

and from the uniqueness of the limit, (96) yields

$$
\mathcal{D}_{b^{-}}^{\alpha}\left(\beta \mathbb{D}_{a^{+}}^{\alpha} y_{n}\right) \rightarrow \mathcal{D}_{b^{-}}^{\alpha}\left(\beta \mathbb{D}_{a^{+}}^{\alpha} y\right) \text { weakly in } L^{2}\left(0, T ; L^{2}(a, b)\right) .
$$

From lemma 3.4, we have that $y \in C\left([0, T] ; L^{2}(a, b)\right)$ and consequently, $y(0)$ and $y(T)$ exists and belong to $L^{2}(a, b)$. We have for all $\phi \in C_{c}^{\infty}\left([0, T] ; L^{2}(a, b)\right)$

$$
\int_{0}^{T} \int_{a}^{b} \partial_{t} y_{n} \phi d x d t=-\int_{0}^{T} \int_{a}^{b} y_{n} \partial_{t} \phi d x d t
$$


Taking the limits yields

$$
\lim _{n \rightarrow \infty} \int_{0}^{T} \int_{a}^{b} \partial_{t} y_{n} \phi d x d t=-\int_{0}^{T} \int_{a}^{b} y \partial_{t} \phi d x d t
$$

which after integrate gives

$$
\lim _{n \rightarrow \infty} \int_{0}^{T} \int_{a}^{b} \partial_{t} y_{n} \phi d x d t=\int_{0}^{T} \int_{a}^{b} \partial_{t} y \phi d x d t .
$$

We obtain

$$
\partial_{t} y_{n} \rightarrow \partial_{t} y \text { weakly in } L^{2}\left(0, T ; L^{2}(a, b)\right)
$$

Passing to the limit in (93) 1 while using (98), (97) and (95c), we deduce that

$$
\partial_{t} y+\mathcal{D}_{b^{-}}^{\alpha}\left(\beta \mathbb{D}_{a^{+}}^{\alpha} y\right)+q y=f \text { in }(a, b) \times(0, T)
$$

Now, if we multiply the first equation in (93) by $\phi \in \mathcal{C}_{c}^{1}(0, T ;(a, b))$ such that $I_{a^{+}}^{1-\alpha} \phi\left(a^{+}\right)=0$ and $I_{a^{+}}^{1-\alpha} \phi\left(b^{-}\right) \neq 0$, then integrate by parts over $(a, b) \times(0, T)$ while using (18), we have that

$$
\begin{aligned}
\int_{0}^{T} \int_{a}^{b} f \phi d s d t & =\int_{0}^{T} \int_{a}^{b}\left(\partial_{t} y_{n}+\mathcal{D}_{b^{-}}^{\alpha}\left(\beta \mathbb{D}_{a^{+}}^{\alpha} y_{n}\right)+q y_{n}\right) \phi d s d t \\
& =-\int_{0}^{T} v_{n} I_{a^{+}}^{1-\alpha}(\phi)\left(b^{-}\right) d t+\int_{0}^{T} \int_{a}^{b} \partial_{t} y_{n} \phi d s d t \\
& +\int_{0}^{T} \int_{a}^{b}\left(\beta \mathbb{D}_{a^{+}}^{\alpha} y_{n}\right)\left(\mathbb{D}_{a^{+}}^{\alpha} \phi\right) d s d t+\int_{0}^{T} \int_{a}^{b} q y_{n} \phi d s d t .
\end{aligned}
$$

Passing to the limit in this latter identity while using (95a), (95c), (95d) and (98), we obtain that

$$
\begin{aligned}
\int_{0}^{T} \int_{a}^{b} f \phi d s d t= & -\int_{0}^{T} u I_{a^{+}}^{1-\alpha}(\phi)\left(b^{-}\right) d t+\int_{0}^{T} \int_{a}^{b} \partial_{t} y \phi d s d t \\
+ & \int_{0}^{T} \int_{a}^{b}\left(\beta \mathbb{D}_{a^{+}}^{\alpha} y\right)\left(\mathbb{D}_{a^{+}}^{\alpha} \phi\right) d s d t+\int_{0}^{T} \int_{a}^{b} q y \phi d s d t \\
& \forall \phi \in \mathcal{C}_{c}^{1}(0, T ;(a, b)) \text { such that } I_{a^{+}}^{1-\alpha} \phi\left(a^{+}\right)=0 \\
& \text { and } I_{a^{+}}^{1-\alpha} \phi\left(b^{-}\right) \neq 0 .
\end{aligned}
$$

On the other hand, $y_{n}(t)$ being in $V_{0}$ which is closed in $\mathcal{V}$, we have that $y \in \mathcal{V}$. It then follows from Lemma 2.3 that $I_{a^{+}}^{1-\alpha} y$ and $\left(\beta \mathbb{D}_{a^{+}}^{\alpha} y\right)$ belong to $\mathcal{C}([0, T] ;[a, b])$. So, if we integrate by parts the first integral in the right side of (100), we get 


$$
\begin{aligned}
\int_{0}^{T} \int_{a}^{b} f \phi d s d t= & \int_{0}^{T}\left(\left(\beta \mathbb{D}_{a^{+}}^{\alpha} y\right)\left(b^{-}\right)-u\right) I_{a^{+}}^{1-\alpha}(\phi)\left(b^{-}\right) d t+\int_{0}^{T} \int_{a}^{b} \partial_{t} y \phi d s d t \\
+ & \int_{0}^{T} \int_{a}^{b} \mathcal{D}_{b^{-}}^{\alpha}\left(\beta \mathbb{D}_{a^{+}}^{\alpha} y\right) \phi d s d t+\int_{0}^{T} \int_{a}^{b} q y \phi d s d t \\
& \forall \phi \in \mathcal{C}_{c}^{1}(0, T ;(a, b)) \text { such that } I_{a^{+}}^{1-\alpha} \phi\left(a^{+}\right)=0 \\
& \text { and } I_{a^{+}}^{1-\alpha} \phi\left(b^{-}\right) \neq 0,
\end{aligned}
$$

which in view of (99) gives

$$
\begin{gathered}
0=\left(\left(\beta \mathbb{D}_{a^{+}}^{\alpha} y\right)\left(b^{-}\right)-u\right) I_{a^{+}}^{1-\alpha}(\phi)\left(b^{-}\right) \forall \phi \in \mathcal{C}_{c}^{1}(0, T(a, b)) \\
\text { such that } I_{a^{+}}^{1-\alpha} \phi\left(a^{+}\right)=0 \text { and } I_{a^{+}}^{1-\alpha} \phi\left(b^{-}\right) \neq 0
\end{gathered}
$$

Hence, we deduce from (101) that

$$
\left(\beta \mathbb{D}_{a^{+}}^{\alpha} y\right)\left(b^{-}, t\right)=u
$$

and From (95g) and (93) 2 , we have that

$$
I_{a^{+}}^{1-\alpha} y\left(a^{+}, t\right)=0
$$

If we multiply the first equation in (93) by $\phi \in \mathcal{C}^{1}([0, T] ;(a, b))$ such that $I_{a^{+}}^{1-\alpha} \phi\left(a^{+}\right)=I_{a^{+}}^{1-\alpha} \phi\left(b^{-}\right)=0$ and $\phi(T)=0$, then integrate by parts over $(a, b) \times(0, T)$ while using $(18)$, we have that

$$
\begin{aligned}
\int_{0}^{T} \int_{a}^{b} f \phi d s d t & =\int_{0}^{T} \int_{a}^{b}\left(\partial_{t} y_{n}+\mathcal{D}_{b^{-}}^{\alpha}\left(\beta \mathbb{D}_{a^{+}}^{\alpha} y_{n}\right)+q y_{n}\right) \phi d s d t \\
& =-\int_{a}^{b} y_{n}(0) \phi(0) d s-\int_{0}^{T} \int_{a}^{b} y_{n} \partial_{t} \phi d s d t \\
& +\int_{0}^{T} \int_{a}^{b}\left(\beta \mathbb{D}_{a^{+}}^{\alpha} y_{n}\right)\left(\mathbb{D}_{a^{+}}^{\alpha} \phi\right) d s d t+\int_{0}^{T} \int_{a}^{b} q y_{n} \phi d s d t .
\end{aligned}
$$

Passing to the limits in this later identity, we get

$$
\begin{aligned}
\int_{0}^{T} \int_{a}^{b} f \phi d s d t= & -\int_{a}^{b} y^{0} \phi(0) d s-\int_{0}^{T} \int_{a}^{b} y \partial_{t} \phi d s d t \\
+ & \int_{0}^{T} \int_{a}^{b}\left(\beta \mathbb{D}_{a^{+}}^{\alpha} y\right)\left(\mathbb{D}_{a^{+}}^{\alpha} \phi\right) d s d t+\int_{0}^{T} \int_{a}^{b} q y \phi d s d t \\
& \forall \phi \in \mathcal{C}^{1}([0, T] ;(a, b)) \text { such that } \phi(T)=0 \\
& \text { and } I_{a^{+}}^{1-\alpha} \phi\left(a^{+}\right)=I_{a^{+}}^{1-\alpha} \phi\left(b^{-}\right)=0 .
\end{aligned}
$$


By integrate the right hand side of this later identity, we obtain

$$
\begin{aligned}
\int_{0}^{T} \int_{a}^{b} f \phi d s d t= & -\int_{a}^{b} y^{0} \phi(0) d s+\int_{a}^{b} y(0) \phi(0) d s \\
+ & \int_{0}^{T} \int_{a}^{b}\left(\partial_{t} y+\mathcal{D}_{b^{-}}^{\alpha}\left(\beta \mathbb{D}_{a^{+}}^{\alpha} y\right)+q y\right) \phi d s d t \\
& \forall \phi \in \mathcal{C}^{1}([0, T] ;(a, b)) \text { such that } \phi(T)=0 \\
& \text { and } I_{a^{+}}^{1-\alpha} \phi\left(a^{+}\right)=I_{a^{+}}^{1-\alpha} \phi\left(b^{-}\right)=0,
\end{aligned}
$$

which in view of (99) gives

$$
\begin{aligned}
0= & \int_{a}^{b}\left[y(0)-y^{0}\right] \phi(0) d s \\
& \forall \phi \in \mathcal{C}^{1}([0, T] ;(a, b)) \text { such that } \phi(T)=0 \\
& \text { and } I_{a^{+}}^{1-\alpha} \phi\left(a^{+}\right)=I_{a^{+}}^{1-\alpha} \phi\left(b^{-}\right)=0 .
\end{aligned}
$$

Thus, we deduce

$$
y(0)=y^{0} .
$$

Hence, (99), (102), (103) and (104) allow us to say that $(u, y) \in L^{2}(0, T) \times W_{0}(0, T)$ is solution of (59). It follows from the semi continuity of the functional $J$ that

$$
J(u) \leq \lim \inf _{v \in \mathcal{U}_{a d}} J\left(v_{n}\right)=\min _{v \in \mathcal{U}_{a d}} J(v) .
$$

This means that

$$
J(u)=\min _{v \in \mathcal{U}_{a d}} J(v)
$$

The uniqueness of the optimal $u$ follows from the strict convexity of $J$.

We proceed now with sufficient optimality conditions.

Theorem 3.5. Let $u$ be a solution of (90). Then there exists $p \in L^{2}(0, T ; V)$ such that $(u, y=y(u), p)$ satisfies

$$
\begin{aligned}
& \left\{\begin{array}{lll}
\partial_{t} y+\mathcal{D}_{b^{-}}^{\alpha} \beta \mathbb{D}_{a^{+}}^{\alpha} y+q y & =f \quad \text { in }(a, b) \times(0, T), \\
I_{a^{+}}^{1-\alpha} y\left(a^{+}, t\right) & =0 \quad \text { in }(0, T), \\
\beta(b) \mathbb{D}_{a^{+}}^{\alpha}\left(b^{-}, t\right) & =u \text { in }(0, T), \\
y(x, 0) & =y^{0} \text { in }(a, b),
\end{array}\right.
\end{aligned}
$$

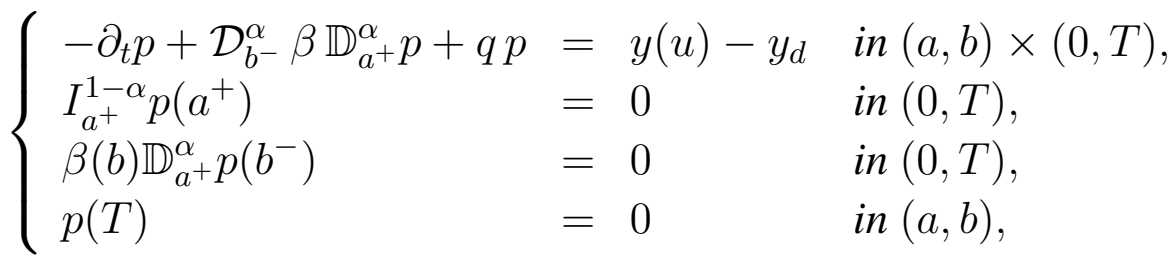

and

$$
\int_{0}^{T}\left[\gamma u+\left(I_{a^{+}}^{1-\alpha} p\right)\left(b^{-}\right)\right] v d t \geq 0 \quad \forall v \in \mathcal{U}_{a d}
$$


Proof. Relation (99), (102) and (103) give (105). To prove (106) and (107), we take directional derivative with respect to $u$ in the direction $v$. We have

$$
\lim _{\lambda \rightarrow 0} \frac{J(u+\lambda v)-J(u)}{\lambda} \geq 0 \quad \forall v \in \mathcal{U}_{a d},
$$

which after a short calculation gives

$$
\int_{0}^{T} \int_{a}^{b} z\left(y(u)-y_{d}\right) d x d t+\gamma \int_{0}^{T} u . v d t \geq 0 \quad \forall v \in \mathcal{U}_{a d}
$$

where $z$ is solution of

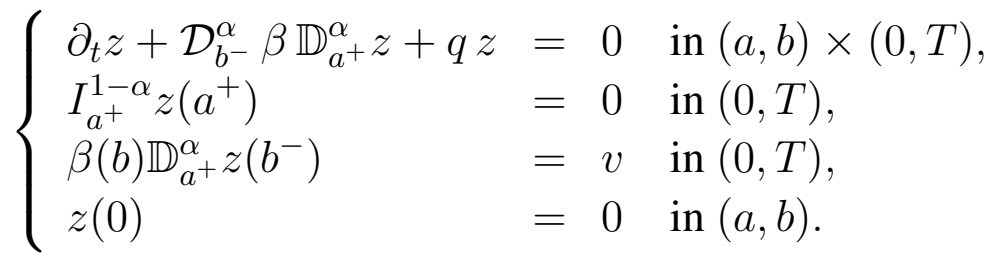

To interpret (108), we consider the adjoint state equation:

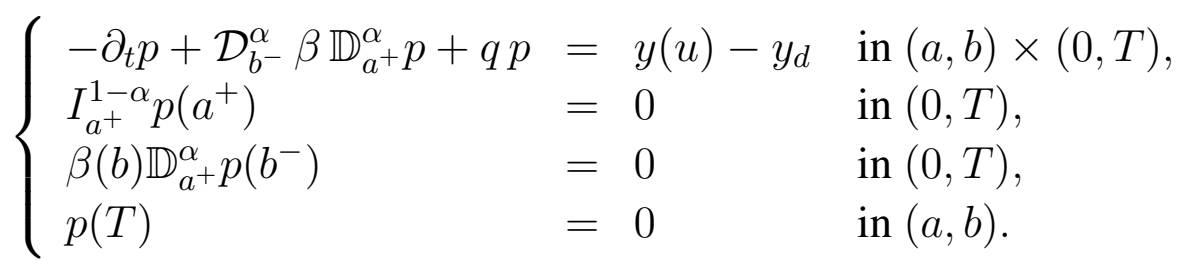

Since $y(u)-y_{d} \in L^{2}\left(0, T ; L^{2}(a, b)\right)$, applying lemma 3.3, we deduce that problem (110) has a unique solution in $W(0, T)$. It then follows from (108) that

$$
\int_{0}^{T}\left[\gamma u+\left(I_{a^{+}}^{1-\alpha} p\right)\left(b^{-}\right)\right] v d t \geq 0 \quad \forall v \in \mathcal{U}_{a d}
$$

4. Optimal boundary control for a parabolic fractional Sturm-Liouville problem on a star graph

\subsection{Existence results of boundary value fractional diffusion Sturm-Liouville equations}

We now consider fractional Sturm-Liouville problems on a general star graph.

$$
\begin{array}{ll}
\partial_{t} y^{i}+\mathcal{D}_{b_{i}^{-}}^{\alpha}\left(\beta^{i} \mathbb{D}_{a^{+}}^{\alpha} y^{i}\right)+q^{i} y^{i} & =f^{i}, \text { in }\left(a, b_{i}\right) \times(0, T), i=1, \ldots, n, \\
I_{a^{+}}^{1-\alpha} y^{i}\left(a^{+}\right) & =I_{a^{+}}^{1-\alpha} y^{j}\left(a^{+}\right) \text {in }(0, T), i \neq j=1, \ldots, n, \\
\sum_{i=1}^{n} \beta^{i}(a) \mathbb{D}_{a^{+}}^{\alpha} y^{i}\left(a^{+}\right) & =0, \quad \text { in }(0, T), \\
I_{a^{+}}^{1-\alpha} y^{1}\left(b_{1}^{-}\right) & =0, \quad \text { in }(0, T), \\
I_{a^{+}}^{1-\alpha} y^{i}\left(b_{i}^{-}\right) & =u_{i}, \quad \text { in }(0, T), i=2, \ldots, m \\
\beta^{i}\left(b_{i}\right) \mathbb{D}_{a^{+}}^{\alpha} y^{i}\left(b_{i}^{-}\right) & =v_{i}, \quad \text { in }(0, T), i=m+1, \ldots, n, \\
y^{i}(0) & =y^{0, i}, \text { in }\left(a, b_{i}\right), i=1, \ldots, n .
\end{array}
$$

We denote $\mathcal{H}^{\alpha}:=\prod_{i=1}^{n} H_{a^{+}}^{\alpha}\left(a, b_{i}\right)$ and by $\mathcal{V}_{i}, i=1, \ldots, n$ the set

$$
\mathcal{V}_{i}=\left\{y^{i} \in H_{a^{+}}^{\alpha}\left(a, b_{i}\right), \quad \mathcal{D}_{b^{-}}^{\alpha}\left(\beta^{i} \mathbb{D}_{a^{+}}^{\alpha} y^{i}\right) \in H_{b^{-}}^{1-\alpha}\left(a, b_{i}\right)\right\}
$$


If we multiply (111) by a function $\phi=\left(\phi^{i}\right)_{i=1, \ldots, n} \in \prod_{i=1}^{n} \mathcal{V}_{i}$ and integrate by parts over $\left(a, b_{i}\right)$, using (18), we have

$$
\begin{aligned}
\sum_{i=1}^{n} \int_{a}^{b_{i}} f^{i}(s) \phi^{i}(s) d s & =\sum_{i=1}^{n} \int_{a}^{b_{i}}\left(\beta^{i}(s) \mathbb{D}_{a^{+}}^{\alpha} y^{i}(s) \mathbb{D}_{a^{+}}^{\alpha} \phi^{i}(s)+q^{i}(s) y^{i}(s) \phi^{i}(s)\right) d s \\
& +\sum_{i=1}^{n} \int_{a}^{b_{i}} \partial_{t} y^{i}(s) \phi^{i}(s) d s-\sum_{i=1}^{n}\left(\beta^{i}\left(b_{i}\right) \mathbb{D}_{a^{+}}^{\alpha} y^{i}\left(b_{i}^{-}\right) I_{a^{+}}^{1-\alpha} \phi^{i}\left(b_{i}^{-}\right)\right) \\
& +\sum_{i=1}^{n}\left(\beta^{i}(a) \mathbb{D}_{a^{+}}^{\alpha} y^{i}\left(a^{+}\right) I_{a^{+}}^{1-\alpha} \phi^{i}\left(a^{+}\right)\right)
\end{aligned}
$$

which if we assume that $\phi$ verifies the nodal conditions gives,

$$
\begin{aligned}
& \sum_{i=1}^{n} \int_{a}^{b_{i}}\left(\partial_{t} y^{i}(s) \phi^{i}(s)+\beta^{i}(s) \mathbb{D}_{a^{+}}^{\alpha} y^{i}(s) \mathbb{D}_{a^{+}}^{\alpha} \phi^{i}(s)+q^{i}(s) y^{i}(s) \phi^{i}(s)\right) d s= \\
& \sum_{i=m+1}^{n} v_{i} I_{a^{+}}^{1-\alpha} \phi^{i}\left(b_{i}^{-}\right)+\sum_{i=1}^{n} \int_{a}^{b_{i}} f^{i}(s) \phi^{i}(s) d s .
\end{aligned}
$$

We define our operator $\mathcal{A}$ as

$$
(\mathcal{A} y)_{i=1, \ldots, n}=\left(\partial_{t} y^{i}+\mathcal{D}_{b^{-}}^{\alpha}\left(\beta^{i} \mathbb{D}_{a^{+}}^{\alpha} y^{i}\right)+q^{i} y^{i}\right)_{i=1, \ldots, n}
$$

and

$$
\begin{aligned}
D(\mathcal{A})= & \left\{\left(y^{i}\right)_{i} \in \prod_{i=1}^{n} \mathcal{V}_{i}, \sum_{i=1}^{n} \beta^{i}(a) \mathbb{D}_{a^{+}}^{\alpha} y^{i}\left(a^{+}\right)=0,\right. \\
& I_{a^{+}}^{1-\alpha} y^{i}\left(a^{+}\right)=I_{a^{+}}^{1-\alpha} y^{j}\left(a^{+}\right), i \neq j, i, j=1, \ldots, n, \\
& I_{b_{i}^{-}}^{1-\alpha} y^{i}\left(b_{i}^{-}\right)=0, i=1, \ldots, m \\
& \left.\beta^{i}\left(b_{i}\right) \mathbb{D}_{a^{+}}^{\alpha} y^{i}\left(b_{i}^{-}\right)=0, i=m+1, \ldots, n\right\} .
\end{aligned}
$$

For the weak formulation, we define the space $\mathbb{V}$ by

$$
\begin{aligned}
\mathbb{V}= & \left\{\phi \in \prod_{i=1}^{n} \mathcal{V}_{i}: I_{a^{+}}^{1-\alpha} \phi^{i}\left(a^{+}\right)=I_{a^{+}}^{1-\alpha} \phi^{j}\left(a^{+}\right), i \neq j=1, \ldots, n,\right. \\
& \left.I_{b_{i}^{+}}^{1-\alpha} \phi^{i}\left(b_{i}^{-}\right)=0, i=1, \ldots, m\right\} .
\end{aligned}
$$

Endowed with the norm

$$
\|\phi\|_{\mathbb{V}}^{2}:=\sum_{i=1}^{n}\left\|\phi^{i}\right\|_{H_{a^{+}}^{\alpha}\left(a, b_{i}\right)}^{2}
$$

the space $\mathbb{V}$ is an Hilbert space. We thus obtain for $y \in D(\mathcal{A})$

$$
\begin{aligned}
\sum_{i=1}^{n} \int_{a}^{b_{i}} \mathcal{A} y(x) \phi(x) d x & =\sum_{i=1}^{n} \int_{a}^{b_{i}} \partial_{t} y(x) d x+\sum_{i=1}^{n} \int_{a}^{b_{i}} \beta^{i}(x) \mathbb{D}_{a^{+}}^{\alpha} y^{i}(x) \mathbb{D}_{a^{+}}^{\alpha} \phi^{i}(x) d x \\
& +\sum_{i=1}^{n} \int_{a}^{b_{i}} q^{i}(x) y^{i}(x) \phi^{i}(x) d x, \quad \forall \phi \in \mathbb{V} .
\end{aligned}
$$


Corollary 4.1. The Hilbert space $\mathbb{V}$ is separable as a subset of separable space $H_{a^{+}}^{\alpha} \times \cdots \times H_{a^{+}}^{\alpha}$.

From now on, we set $H:=\prod_{i=1}^{n} L^{2}\left(a, b_{i}\right)$ and we endow $H$ with the norm

$$
\|f\|_{H}^{2}=\sum_{i=1}^{n}\left\|f^{i}\right\|_{L^{2}\left(a, b_{i}\right)}^{2}, \quad \forall f=\left(f_{i}\right) \in H .
$$

Theorem 4.1. Let $q^{i} \in L^{\infty}\left(a, b_{i}\right), i=1, \ldots, n$ and $\beta^{i} \in \mathcal{C}\left(\left[a, b_{i}\right]\right), i=1, \ldots, n$ be such that $\beta^{i}>\beta_{0}^{i}>$ 0 and $q^{i} \geq q_{0}^{i}>0$. Then there exists a unique weak solution $y \in C([0, T] ; \mathbb{V}) \cap H^{1}(0, T ; \mathbb{V})$ solution of the problem (111).

Proof. In order to treat (111) in the framework of Galerkin approximation while using the Lax-Milgram Lemma, we need to shift the non-homogeneous Dirichlet data to the state equation and Neumann-data. To this end, we define functions

$$
w^{i}(x, t):= \begin{cases}u_{i} \frac{(x-a)^{\nu}}{\left(b_{i}-a\right)^{\nu-\alpha+1}} \frac{\Gamma(\nu-\alpha+2)}{\Gamma(\nu+1)}, & i=1, \ldots, m, \quad \nu>\frac{2 \alpha+1}{2} \\ 0 & i=m+1, \ldots, n .\end{cases}
$$

Then,

$$
\begin{aligned}
& I_{a}^{1-\alpha} w^{i}\left(a^{+}, t\right)=0, \quad I_{a}^{1-\alpha} w^{i}\left(b_{i}^{-}, t\right)=u_{i}, i=1, \ldots, m, \\
& \mathbb{D}_{a^{+}}^{\alpha} w^{i}(x, t)=\frac{d}{d x} I_{a^{+}}^{1-\alpha} w^{i}(x, t)=u_{i} \frac{\nu-\alpha+1}{\left(b_{i}-a\right)^{\nu-\alpha+1}}(x-a)^{\nu-\alpha}, \\
& \mathbb{D}_{a^{+}}^{\alpha} w^{i}\left(a^{+}, t\right)=0, \nu>\frac{2 \alpha+1}{2}, \quad i=1, \ldots, m \\
& \partial_{t} w^{i}(x, t)+\mathcal{D}_{b_{i}^{-}}^{\alpha}\left(\beta^{i} \mathbb{D}_{a^{+}}^{\alpha} w^{i}\right)(x, t)+q^{i}(x) w^{i}(x, t)=: u_{i} g_{i}(x, t)= \\
& \quad u_{i} \frac{\nu-\alpha+1}{\left(b_{i}-a\right)^{\nu-\alpha+1}}\left(\mathcal{D}_{b_{i}^{-}}^{\alpha}\left(\beta(\cdot)(\cdot-a)^{\nu-\alpha}(x)+q^{i}(x)(x-a)^{\nu} \frac{\Gamma(\nu-\alpha+1)}{\Gamma(\nu+1)}\right)\right. \\
& i=1, \ldots, m .
\end{aligned}
$$

Remark 3. Note that we have $\partial_{t} w^{i}+\mathcal{D}_{b_{i}^{-}}^{\alpha}\left(\beta^{i} \mathbb{D}_{a^{+}}^{\alpha} w^{i}\right)+q^{i}(x) w^{i} \in L^{2}\left(0, T ;\left(a, b_{i}\right)\right), i=1, \cdots, n$. Indeed,

1) $\partial_{t} w^{i} \in L^{2}\left(0, T ;\left(a, b_{i}\right)\right)$ and $q^{i} w^{i} \in L^{2}\left(0, T ;\left(a, b_{i}\right)\right)$ because $w^{i} \in L^{2}\left(0, T ;\left(a, b_{i}\right)\right)$ and $q^{i} \in$ $C\left(\left[a, b_{i}\right]\right)$.

2) Set $h_{i}(x)=\beta^{i}(x)(x-a)^{\nu-\alpha}$. Then under the assumptions on $\beta^{i}$ we have that $h_{i}^{\prime} \in L^{2}\left(a, b_{i}\right)$. Therefore, it follows from Lemma 2.1 that $I_{b_{i}^{-}}^{1-\alpha} h_{i}^{\prime} \in L^{2}\left(0, T ;\left(a ; b_{i}\right)\right)$. Observing that

$$
\begin{aligned}
\mathcal{D}_{b_{i}^{-}}^{\alpha}\left(\beta^{i} \mathbb{D}_{a^{+}}^{\alpha} w^{i}\right)(x) & =u_{i} \frac{\nu-\alpha+1}{\left(b_{i}-a\right)^{\nu-\alpha+1}} \mathcal{D}_{b_{i}^{-}}^{\alpha}\left(\beta(\cdot)(\cdot-a)^{\nu-\alpha}(x)\right. \\
& =u_{i} \frac{\nu-\alpha+1}{\left(b_{i}-a\right)^{\nu-\alpha+1}} I_{b_{i}^{-}}^{1-\alpha} h_{i}^{\prime}(x),
\end{aligned}
$$

we deduce from 1) and 2) that $\partial_{t} w^{i}+\mathcal{D}_{b_{i}^{-}}^{\alpha}\left(\beta^{i} \mathbb{D}_{a^{+}}^{\alpha} w^{i}\right)+q^{i}(x) w^{i} \in L^{2}\left(0, T ;\left(a, b_{i}\right)\right), i=1, \cdots, n$. 
We define the new right hand side

$$
F^{i}\left(x, t ; u_{i}\right):= \begin{cases}f^{i}(x, t)-u_{i} g_{i}(x, t) & i=1, \ldots, m \\ f^{i}(x, t) & i=m+1, \ldots, n\end{cases}
$$

and decompose the solution $y$ to (111) as

$$
y^{i}(x, t):=w^{i}(x, t)+\hat{y}^{i}(x, t), i=1, \ldots, n .
$$

where now $\hat{y}$ solves

$$
\begin{aligned}
\partial_{t} \hat{y}^{i}+\mathcal{D}_{b_{i}^{-}}^{\alpha}\left(\beta^{i} \mathbb{D}_{a^{+}}^{\alpha} \hat{y}^{i}\right)+q^{i} \hat{y}^{i} & =F^{i}\left(x, t ; u_{i}\right), \text { in }\left(a, b_{i}\right) \times(0, T), i=1, \ldots, n, \\
I_{a^{+}}^{1-\alpha} \hat{y}^{i}\left(a^{+}\right) & =I_{a^{+}}^{1-\alpha} \hat{y}^{j}\left(a^{+}\right) \text {in }(0, T), i \neq j=1, \ldots, n, \\
\sum_{i=1}^{n} \beta^{i}(a) \mathbb{D}_{a^{+}}^{\alpha} \hat{y}^{i}\left(a^{+}\right) & =0, \quad \text { in }(0, T), \\
I_{a^{+}}^{1-\alpha} \hat{y}^{i}\left(b_{i}^{-}\right) & =0, \quad \text { in }(0, T), i=1, \ldots, m \\
\beta^{i}\left(b_{i}\right) \mathbb{D}_{a^{+}}^{\alpha} \hat{y}^{i}\left(b_{i}^{-}\right) & =v_{i}, \quad \text { in }(0, T), i=m+1, \ldots, n, \\
\hat{y}^{i}(0) & =y^{0, i}, \text { in }\left(a, b_{i}\right), i=1, \ldots, n .
\end{aligned}
$$

Now, (124) is a boundary value problem with homogeneous Dirichlet data, where the controls now appear in the fractional Neumann conditions, only, and in the distributed right hand side. As shown below, these data are included in the linear form $L$. For convenience, we replace $\hat{y}^{i}$ by $y^{i}$. At the end of the proof, we have to add $w^{i}$.

For any $y, \phi \in \mathbb{V}$, we respectively defined on $\mathbb{V} \times \mathbb{V}$ and on $\mathbb{V}$, the bilinear functional $\pi(.,$.$) and the$ linear functional $L($.$) by :$

$$
\begin{aligned}
& \pi(y, \phi)=\sum_{i=1}^{n} \int_{a}^{b_{i}} \beta^{i}(x) \mathbb{D}_{a^{+}}^{\alpha} y^{i}(x) \mathbb{D}_{a^{+}}^{\alpha} \phi^{i}(x) d x+\sum_{i=1}^{n} \int_{a}^{b_{i}} q^{i}(x) y^{i}(x) \phi^{i}(x) d x . \\
& L(\phi)=\sum_{i=m+1}^{n} v_{i} I_{a^{+}}^{1-\alpha} \phi^{i}\left(b_{i}^{-}\right)+\sum_{i=1}^{n} \int_{a}^{b_{i}} F^{i}\left(s ; u_{i}\right) \phi^{i}(s) d s .
\end{aligned}
$$

The bilinear functional $\pi(.,$.$) is continuous and coercive and the linear functional L($.$) continuous. In-$ deed, using the Cauchy-Schwarz inequality and the fact that $\beta^{i} \in L^{\infty}\left(a, b_{i}\right), q \in \mathcal{C}\left(\left[a, b_{i}\right]\right)$ such that $\beta^{i}>\beta_{0}^{i}>0$ and $q^{i}>q_{0}^{i}>0$, we have

$$
\begin{aligned}
|\pi(y, \phi)| & \leq\left(\sum_{j=1}^{n}\left\|\beta^{j}\right\|_{\infty}\right) \sum_{i=1}^{n}\left(\left\|\mathbb{D}_{a^{+}}^{\alpha} y^{i}\right\|_{L^{2}\left(a, b_{i}\right)}\left\|\mathbb{D}_{a^{+}}^{\alpha} \phi^{i}\right\|_{L^{2}\left(a, b_{i}\right)}\right) \\
& +\left(\sum_{j=1}^{n}\left\|q^{j}\right\|_{\infty}\right) \sum_{i=1}^{n}\left(\left\|y^{i}\right\|_{L^{2}\left(a, b_{i}\right)}\left\|\phi^{i}\right\|_{L^{2}\left(a, b_{i}\right)}\right) \\
\leq & \sum_{j=1}^{n}\left(\left\|\beta^{j}\right\|_{\infty}+\left\|q^{j}\right\|_{\infty}\right) \sum_{i=1}^{n}\left(\left\|\mathbb{D}_{a^{+}}^{\alpha} y^{i}\right\|_{L^{2}\left(a, b_{i}\right)}\left\|\mathbb{D}_{a^{+}}^{\alpha} \phi^{i}\right\|_{L^{2}\left(a, b_{i}\right)}\right) \\
& +\sum_{j=1}^{n}\left(\left\|\beta^{j}\right\|_{\infty}+\left\|q^{j}\right\|_{\infty}\right) \sum_{i=1}^{n}\left(\left\|y^{i}\right\|_{L^{2}\left(a, b_{i}\right)}\left\|\phi^{i}\right\|_{L^{2}\left(a, b_{i}\right)}\right) \\
\leq & \sum_{j=1}^{n}\left(\left\|\beta^{j}\right\|_{\infty}+\left\|q^{j}\right\|_{\infty}\right) \sum_{i=1}^{n}\left(\left\|\mathbb{D}_{a^{+}}^{\alpha} y^{i}\right\|_{L^{2}\left(a, b_{i}\right)}^{2}+\left\|y^{i}\right\|_{L^{2}\left(a, b_{i}\right)}^{2}\right)^{1 / 2} \times \\
& \left(\left\|\mathbb{D}_{a^{+}}^{\alpha} \phi^{i}\right\|_{L^{2}\left(a, b_{i}\right)}^{2}+\left\|\phi^{i}\right\|_{L^{2}\left(a, b_{i}\right)}^{2}\right)^{1 / 2}
\end{aligned}
$$




$$
\begin{aligned}
& \leq \sum_{j=1}^{n}\left(\left\|\beta^{j}\right\|_{\infty}+\left\|q^{j}\right\|_{\infty}\right)\left(\sum_{i=1}^{n}\left\|y^{i}\right\|_{H_{a^{+}}^{\alpha}\left(a, b_{i}\right)}^{2}\right)^{1 / 2}\left(\sum_{i=1}^{n}\left\|\phi^{i}\right\|_{H_{a^{+}}^{\alpha}\left(a, b_{i}\right)}^{2}\right)^{1 / 2} \\
& \leq \sum_{j=1}^{n}\left(\left\|\beta^{j}\right\|_{\infty}+\left\|q^{j}\right\|_{\infty}\right)\|y\|_{\mathbb{V}}\|\phi\|_{\mathbb{V} .}
\end{aligned}
$$

This means that the bilinear form $\pi(.,$.$) is continuous on \mathbb{V} \times \mathbb{V}$. The bilinear form $\pi(.,$.$) is coercive on$ $\mathbb{V}$ because

$$
\begin{aligned}
\pi(y, y) & =\sum_{i=1}^{n} \int_{a}^{b_{i}} \beta^{i}(x)\left|\mathbb{D}_{a^{+}}^{\alpha} y^{i}(x)\right|^{2} d x+\sum_{i=1}^{n} \int_{a}^{b_{i}} q^{i}(x)\left|y^{i}(x)\right|^{2} d x \\
& \geq \sum_{i=1}^{n} \beta_{0}^{i} \int_{a}^{b_{i}}\left|\mathbb{D}_{a^{+}}^{\alpha} y^{i}(x)\right|^{2} d x+\sum_{i=1}^{n} q_{0}^{i} \int_{a}^{b_{i}}\left|y^{i}(x)\right|^{2} d x \\
& \geq \min \left(\min _{i}\left(\beta_{0}^{i}\right), \min _{i}\left(q_{0}^{i}\right)\right) \sum_{i=1}^{n}\left[\left\|\mathbb{D}_{a^{+}}^{\alpha} y^{i}\right\|_{L^{2}\left(a, b_{i}\right)}^{2}+\left\|y^{i}\right\|_{L^{2}\left(a, b_{i}\right)}^{2}\right] \\
& \geq \min \left(\min _{i}\left(\beta_{0}^{i}\right), \min _{i}\left(q_{0}^{i}\right)\right)\|y\|_{\mathbb{V}}^{2}
\end{aligned}
$$

Since $\phi^{i} \in H_{a^{+}}^{\alpha}\left(a, b_{i}\right) i=1, \ldots, n$, we have on the one hand that,

$$
\begin{aligned}
\left|I_{a^{+}}^{1-\alpha} \phi^{i}\left(b_{i}^{-}\right)-I_{a^{+}}^{1-\alpha} \phi^{i}\left(a^{+}\right)\right| & =\left|\int_{a}^{b_{i}} \frac{d}{d s} I_{a^{+}}^{1-\alpha} \phi^{i}(s) d s\right| \\
& =\left|\int_{a}^{b_{i}} \mathbb{D}_{a^{+}}^{\alpha} \phi^{i}(s) d s\right| \\
& \leq\left|b_{i}-a\right|^{1 / 2}\left\|\mathbb{D}_{a^{+}}^{\alpha} \phi^{i}\right\|_{L^{2}\left(a, b_{i}\right)} \\
& \leq\left|b_{i}-a\right|^{1 / 2}\left\|\phi^{i}\right\|_{H_{a^{+}}^{\alpha}\left(a, b_{i}\right)} \\
& \leq \mid C\|\phi\|_{\mathbb{V}},
\end{aligned}
$$

and on the other hand that, there exists $C>$ such that,

$$
\begin{aligned}
\left|I_{a^{+}}^{1-\alpha} \phi^{i}\left(a^{+}\right)\right| & \leq\left\|\mid \phi^{i}\right\| \|_{H_{a^{+}}^{\alpha}\left(a, b_{i}\right)} \\
& \leq C_{i}\left\|\phi^{i}\right\|_{H_{a^{+}}^{\alpha}\left(a, b_{i}\right)} \\
& \leq C\|\phi\|_{\mathbb{V},}
\end{aligned}
$$

because of (15). Therefore,

$$
\begin{aligned}
|L(\phi)| & =\left|\sum_{i=m+1}^{m} v_{i} I_{a^{+}}^{1-\alpha} \phi^{i}\left(b_{i}^{-}\right)+\sum_{i=1}^{n} \int_{a}^{b_{i}} F^{i}\left(s ; u_{i}\right) \phi^{i}(s) d s\right| \\
& \leq \sum_{i=m+1}^{n}\left(\left|v_{i} \| I_{a^{+}}^{1-\alpha} \phi^{i}\left(b_{i}^{-}\right)-I_{a^{+}}^{1-\alpha} \phi^{i}\left(a^{+}\right)\right|+\left|v_{i}\right|\left|I_{a^{+}}^{1-\alpha} \phi^{i}\left(a^{+}\right)\right|\right) \\
& +\left|\sum_{i=1}^{n} \int_{a}^{b_{i}} F^{i}\left(s ; u_{i}\right) \phi^{i}(s) d s\right| \\
& \leq\|v\|\left(C^{2}+\|F\|_{H}^{2}\right)^{1 / 2}\|\phi\|_{\mathbb{V}}
\end{aligned}
$$


Equation (124) is therefore equivalent to the following system

$$
\begin{cases}y \in C\left(0, T,\left[a, b_{i}\right]\right) \cap H^{1}(0, T ; \mathbb{V}) & \\ \sum_{i=1}^{n} \frac{d}{d t}\left(y^{i}, \phi\right)+\pi(t ; y, \phi) & =L(\phi), \quad \forall \phi \in \mathbb{V}, \\ y^{i}(0) & =y^{0, i}, \quad i=1, \cdots, n .\end{cases}
$$

For the rest of the proof, we will procede in three steps.

Uniqueness. Let $y_{1}^{i}$ and $y_{2}^{i}$ be two solution of equation (124), $i=1, \cdots, n$. By setting $y^{i}=y_{1}^{i}-y_{2}^{i}, y^{i}$, $i=1, \cdots, n$ satisfy (124) with $F^{i}=0, y^{0, i}=0, v_{i}=0$. Taking the scalar product with $y^{i}(t)$ under the duality between $\mathbb{V}$ and $\mathbb{V}^{*}$, we get

$$
\pi(t ; y(t), y(t))+\sum_{i=1}^{n}\left(\frac{d y^{i}(t)}{d t}, y^{i}(t)\right)=0
$$

Since

$$
\sum_{i=1}^{n} \int_{0}^{T}\left(\frac{d y^{i}(t)}{d t}, y^{i}(t)\right) d t=\frac{1}{2}\|y(T)\|_{H}^{2}
$$

equation (68) becomes

$$
\pi(t ; y(t), y(t))+\frac{1}{2}\|y(T)\|_{H}^{2}=0 .
$$

Using the coercivity of the bilinear functional, we have

$$
\min \left(\min _{i}\left(\beta_{0}^{i}\right), \min _{i}\left(q_{0}^{i}\right)\right) \int_{0}^{T}\|y(t)\|_{\mathbb{V}}^{2} d t+\frac{1}{2}\|y(T)\|_{H}^{2} \leq 0
$$

and thus

$$
y=0 \text {. }
$$

Existence. The space $\mathbb{V}$ is a separable Hilbert space (see corollary 4.1). Therefore there exists a countable set which is dense in $\mathbb{V}$. We may then find a basis $w_{1}, w_{2}, \cdots, w_{m}, \cdots$ in $\mathbb{V}$ in the following sense:

$$
\left\{\begin{array}{l}
w_{i} \in \mathbb{V} \text { for all } i \\
\forall m, w_{1}, w_{2}, \cdots, w_{m} \text { are linearly independent; } \\
\text { the linear combination } \sum_{i=1}^{m} \phi_{j} w_{j}, \phi_{j} \in \mathbb{R} \text { are dense in } \mathbb{V} .
\end{array}\right.
$$

We then find $y_{m}=y_{m}(t)$ "approximate solution of the problem (124) in the form

$$
y_{m}(t)=\sum_{i=1}^{m} g_{i m}(t) w_{i}
$$

where the $g_{i m}$ are chosen such that

$$
\sum_{i=1}^{n}\left(\frac{d y_{m}^{i}(t)}{d t}, w_{j}^{i}\right)+\pi\left(t ; y_{m}(t), w_{j}\right)=\sum_{i=m+1}^{n} v_{i} I_{a^{+}}^{1-\alpha} w_{j}\left(b_{i}^{-}\right)+\sum_{i=1}^{n} \int_{a}^{b_{i}} F^{i}\left(s ; u_{i}\right) w_{j} d s
$$


$1 \leq j \leq m$, and

$$
y_{m}^{i}(0)=y_{0 m}^{i}=\sum_{i=1}^{m} \varepsilon_{k m}^{i} w_{k}, \quad \sum_{k=1}^{m} \varepsilon_{k m} w_{k} \rightarrow y^{0, i} \text { in } L^{2}\left(a, b_{i}\right) \text { as } m \rightarrow \infty,
$$

where

$$
\pi\left(t ; y(t), w_{j}\right)=\sum_{i=1}^{n} \int_{a}^{b_{i}} \beta^{i}(x) \mathbb{D}_{a^{+}}^{\alpha} y^{i}(x) \mathbb{D}_{a^{+}}^{\alpha} w_{j}^{i}(x) d x+\sum_{i=1}^{n} \int_{a}^{b_{i}} q^{i}(x) y^{i}(x) w_{j}^{i}(x) d x .
$$

System (134), (135) is a system of $m$ linear differential equations in $g_{i m}(t)$ of the form

$$
\mathcal{W}_{m} \frac{d g_{m}}{d t}+\mathcal{A}_{m}(t) g_{m}=f_{m}, g_{m}(0)=\left\{\varepsilon_{i m}\right\}
$$

where

$$
\begin{aligned}
& \mathcal{W}_{m}=\sum_{i=1}^{n}\left\|\left(w_{k}^{i}, w_{j}^{i}\right)\right\| ; \quad \mathcal{A}_{m}(t)=\left\|\pi\left(t ; w_{k}, w_{j}\right)\right\|, \\
& g_{m}(t)=\left\{g_{k m}(t)\right\}, \quad f_{m}(t)=\left\{\sum_{i=m+1}^{n} v_{i} I_{a^{+}}^{1-\alpha} w_{j}\left(b_{i}^{-}\right)+\sum_{i=1}^{n} \int_{a}^{b_{i}} F^{i}\left(s ; u_{i}\right) w_{j} d s\right\} .
\end{aligned}
$$

Since $\operatorname{det} \mathcal{W}_{m} \neq 0$, problem (134), (135) admits a unique solution. It remain to show that as $m \rightarrow \infty$, $y_{m} \rightarrow y, y$ being a solution of (124).

Multiplying equation (134) by $g_{j m}(t)$ and summing over $j$, we obtain

$$
\sum_{i=1}^{n}\left(\frac{d y_{m}^{i}}{d t}, y_{m}^{i}\right)+\pi\left(t ; y_{m}, y_{m}\right)=\sum_{i=m+1}^{n} v_{i} I_{a^{+}}^{1-\alpha} y_{m}\left(b_{i}^{-}\right)+\sum_{i=1}^{n} \int_{a}^{b_{i}} F^{i}\left(s ; u_{i}\right) y_{m} d s
$$

that is

$$
\frac{1}{2} \frac{d}{d t}\left\|y_{m}(t)\right\|_{\mathbb{V}}^{2}+\pi\left(t ; y_{m}, y_{m}\right)=\sum_{i=m+1}^{n} v_{i} I_{a^{+}}^{1-\alpha} y_{m}\left(b_{i}^{-}\right)+\sum_{i=1}^{n} \int_{a}^{b_{i}} F^{i}\left(s ; u_{i}\right) y_{m} d s .
$$

Using equation (127) with a slight modification, the coercivity of $\pi$ with $\alpha=\min \left(\min _{i}\left(\beta_{0}^{i}\right), \min _{i}\left(q_{0}^{i}\right)\right)$ and Cauchy Schwarz and Young inequalities, we obtain

$$
\begin{aligned}
& \left\|y_{m}(T)\right\|_{L^{2}(a, b)}^{2}+2 \alpha \int_{0}^{T}\left\|y_{m}(t)\right\|_{\mathbb{V}}^{2} d t \\
& \leq\left\|y_{0 m}\right\|_{L^{2}(a, b)}^{2}+2 \sum_{i=m+1}^{n} v_{i} I_{a^{+}}^{1-\alpha} y_{m}\left(b_{i}^{-}\right)+2 \sum_{i=1}^{n} \int_{a}^{b_{i}} F^{i}\left(s ; u_{i}\right) y_{m} d s \\
& \leq\left\|y_{0 m}\right\|_{L^{2}(a, b)}^{2}+2\|v\|\left(C^{2}+\|F\|_{H}^{2}\right)^{1 / 2}\|y(t)\|_{\mathbb{V}}
\end{aligned}
$$

From (135) we have $\left\|y_{0 m}\right\|_{L^{2}(a, b)} \geq C\left\|y^{0}\right\|_{L^{2}(a, b)}$ and therefore

$$
\int_{0}^{T}\left\|y_{m}(t)\right\|_{V_{0}}^{2} d t \leq C\left(\left\|y^{0}\right\|_{H(a, b)}^{2}+\|F\|_{\mathbb{V}^{*}}^{2}+\|v\|_{H(0, T)}^{2}\right) .
$$


Equation (140) implies that the sequence $y_{m}^{i}$ is bounded in $L^{2}(0, T ; \mathbb{V})$ and we may extract a subsequence $y_{\mu}^{i}$ such that

$$
y_{\mu}^{i} \rightarrow z^{i} \text { weakly in } L^{2}(0, T ; \mathbb{V}), \quad i=1, \cdots, n .
$$

Let $j$ be fixed but arbitrary and let $\mu>j$. Then (134) is valid with $m=\mu$.

Multiply both sides of (134) by

$$
\phi \in \mathbb{V}, \quad \phi^{i}(t) \in C^{1}[0, T], \quad \phi^{i}(T)=0, \quad i=1, \cdots, n,
$$

and integrate over $(0, T)$ while setting $\phi_{j}^{i}=\phi^{i}(t) w_{j}^{i}$, we have

$$
\begin{aligned}
& -\sum_{i=1}^{n} \int_{0}^{T}\left(y_{\mu}^{i},\left(\phi_{j}^{i}\right)^{\prime}\right) d t+\int_{0}^{T} \pi\left(t ; y_{\mu}, \phi_{j}\right) d t=\sum_{i=m+1}^{n} \int_{0}^{T} v_{i} I_{a^{+}}^{1-\alpha} y_{m}\left(b_{i}^{-}\right) d t \\
& +\sum_{i=1}^{n} \int_{0}^{T} \int_{a}^{b_{i}} F^{i}\left(s ; u_{i}\right) y_{m} d s d t+\sum_{i=1}^{n}\left(y_{\mu}^{0, i}, \phi_{j}^{i}(0)\right) .
\end{aligned}
$$

Using (141), we can pass to the limit in (143). We then obtain

$$
\begin{aligned}
& -\sum_{i=1}^{n} \int_{0}^{T}\left(z^{i},\left(\phi_{j}^{i}\right)^{\prime}\right) d t+\int_{0}^{T} \pi\left(t ; z, \phi_{j}\right) d t=\sum_{i=m+1}^{n} \int_{0}^{T} v_{i} I_{a^{+}}^{1-\alpha} \phi_{j}^{i}\left(b_{i}^{-}\right) d t \\
& +\sum_{i=1}^{n} \int_{0}^{T} \int_{a}^{b_{i}} F^{i}\left(s ; u_{i}\right) \phi_{j}^{i} d s d t+\sum_{i=1}^{n}\left(y^{0, i}, \phi_{j}^{i}(0)\right) .
\end{aligned}
$$

Equation (144) is true for any $\phi$ satisfying (142). Therefore we may take $\phi^{i} \in C_{c}(0, T), i=1, \cdots, n$ and hence (144) gives

$$
\sum_{i=1}^{n} \frac{d}{d t}\left(z^{i}(t), w_{j}^{i}\right)+\pi\left(t ; z(t), w_{j}\right)=\sum_{i=m+1}^{n} v_{i} I_{a^{+}}^{1-\alpha} w_{j}^{i}\left(b_{i}^{-}\right)+\sum_{i=1}^{n} \int_{a}^{b_{i}} F^{i}\left(s ; u_{i}\right) w_{j}^{i} d s .
$$

But in (145) $j$ is arbitrary and since finite linear combination of $w_{j}$ are dense in $\mathbb{V}$,we deduce

$$
\sum_{i=1}^{n} \frac{d z^{i}}{d t}+\mathcal{A} z=\sum_{i=1}^{n} F^{i}\left(s ; u_{i}\right)+\sum_{i=m+1}^{n} v_{i} I_{a^{+}}^{1-\alpha} w_{j}^{i}\left(b_{i}^{-}\right), \quad i=1, \cdots, n .
$$

Therefore,

$$
\sum_{i=1}^{n} \frac{d z^{i}}{d t}=\sum_{i=1}^{n} F^{i}\left(s ; u_{i}\right)+\sum_{i=m+1}^{n} v_{i} I_{a^{+}}^{1-\alpha} w_{j}^{i}\left(b_{i}^{-}\right)-\mathcal{A}(t) z \in L^{2}\left(0, T ; \mathbb{V}^{*}\right),
$$

and hence

$$
z^{i} \in C\left(0, T ;\left[a, b_{i}\right]\right) \cap H^{1}(0, T ; \mathbb{V}) .
$$


This allow us to integrate by part in $t$. Thus, taking into account (146) we obtain

$$
\sum_{i=1}^{n}\left(z^{i}(0), w_{j}^{i}\right) \phi^{i}(0)=\sum_{i=1}^{n}\left(y^{0, i}, w_{j}^{i}\right) \phi^{i}(0) \quad \forall j, \forall \phi^{i}, \quad i=1, \cdots, n .
$$

That is $\left(z^{i}(0), w_{j}^{i}\right) \phi(0)=\left(y^{0, i}, w_{j}\right) \phi(0) \forall j$ and thus $z^{i}(0)=y^{0, i}, i=1, \cdots, n$. Hence $z^{i}$ is solution and therefore $z^{i}=y^{i}, i=1, \cdots, n$ is a solution. We may then replace (141) by

$$
y_{m}^{i} \rightarrow y^{i} \text { weakly in } L^{2}(0, T ; \mathbb{V}), \quad i=1, \cdots, n .
$$

The estimate (140) give us

$$
\|y\|_{L^{2}(0, T ; \mathbb{V})}^{2} \leq C\left(\left\|y^{0}\right\|_{H(a, b)}^{2}+\|F\|_{\mathbb{V}^{*}}^{2}+\|v\|_{H(0, T)}^{2}\right) .
$$

Moreover, since

$$
\sum_{i=1}^{n} \frac{d z^{i}}{d t}=\sum_{i=1}^{n} F^{i}\left(s ; u_{i}\right)+\sum_{i=m+1}^{n} v_{i} I_{a^{+}}^{1-\alpha} w_{j}^{i}\left(b_{i}^{-}\right)-\mathcal{A}(t) z,
$$

we have using (151)

$$
\left\|\frac{d y}{d t}\right\|_{L^{2}\left(0, T ; \mathbb{V}^{*}\right)}^{2} \leq C^{\prime}\left(\left\|y^{0}\right\|_{H(a, b)}^{2}+\|F\|_{\mathbb{V}^{*}}^{2}+\|v\|_{H(0, T)}^{2}\right) .
$$

Corollary 4.2. Let $f^{i} \in L^{2}(0, T ; H), y^{0, i} \in H, q^{i} \in L^{\infty}\left(a, b_{i}\right), i=1, \ldots, n$ and $\beta^{i} \in \mathcal{C}\left(\left[a, b_{i}\right]\right), i=$ $1, \ldots, n$ be such that $\beta^{i}>\beta_{0}^{i}>0$ and $q^{i} \geq q_{0}^{i}>0$. Then there exists a unique weak solution $y \in$ $L^{2}(0, T ; \mathbb{V}) \cap H^{1}(0, T, \mathbb{V})$ solution of the following problem

$$
\begin{array}{ll}
\partial_{t} y^{i}+\mathcal{D}_{b_{i}^{-}}^{\alpha}\left(\beta^{i} \mathbb{D}_{a^{+}}^{\alpha} y^{i}\right)+q^{i} y^{i} & =f^{i}, \text { in }\left(a, b_{i}\right) \times(0, T), i=1, \ldots, n, \\
I_{a^{+}}^{1-\alpha} y^{i}\left(a^{+}\right) & =I_{a^{+}}^{1-\alpha} y^{j}\left(a^{+}\right) \text {in }(0, T), i \neq j=1, \ldots, n, \\
\sum_{i=1}^{n} \beta^{i}(a) \mathbb{D}_{a^{+}}^{\alpha} y^{i}\left(a^{+}\right) & =0, \quad \text { in }(0, T), \\
I_{a^{+}}^{1-\alpha} y^{i}\left(b_{i}^{-}\right) & =0, \quad \text { in }(0, T), i=1, \ldots, m \\
\beta^{i}\left(b_{i}\right) \mathbb{D}_{a^{+}}^{\alpha} y^{i}\left(b_{i}^{-}\right) & =0, \quad \text { in }(0, T), i=m+1, \ldots, n, \\
y^{i}(0) & =0, \quad \text { in }\left(a, b_{i}\right), i=1, \ldots, n .
\end{array}
$$

Proof. The proof follows immediatly from the above theorem.

Lemma 4.1. Let $h^{i} \in L^{2}(0, T ; H), y^{0, i} \in H, q^{i} \in L^{\infty}\left(a, b_{i}\right), i=1, \ldots, n$ and $\beta^{i} \in \mathcal{C}\left(\left[a, b_{i}\right]\right), i=$ $1, \ldots, n$ be such that $\beta^{i}>\beta_{0}^{i}>0$ and $q^{i} \geq q_{0}^{i}>0$. Then there exists a unique weak solution $\phi \in$ $L^{2}(0, T ; \mathbb{V}) \cap H^{1}(0, T, \mathbb{V})$ solution of the following adjoint problem

$$
\begin{aligned}
-\partial_{t} \phi^{i}+\mathcal{D}_{b_{i}^{-}}^{\alpha}\left(\beta^{i} \mathbb{D}_{a^{+}}^{\alpha} \phi^{i}\right)+q^{i} \phi^{i} & =h^{i}, \text { in }\left(a, b_{i}\right) \times(0, T), i=1, \ldots, n, \\
I_{a^{+}}^{1-\alpha} \phi^{i}\left(a^{+}\right) & =I_{a^{+}}^{1-\alpha} \phi^{j}\left(a^{+}\right) \text {in }(0, T), i \neq j=1, \ldots, n, \\
\sum_{i=1}^{n} \beta^{i}(a) \mathbb{D}_{a^{+}}^{\alpha} \phi^{i}\left(a^{+}\right) & =0, \quad \text { in }(0, T), \\
I_{a^{+}}^{1-\alpha} \phi^{i}\left(b_{i}^{-}\right) & =0, \quad \text { in }(0, T), i=1, \ldots, m \\
\beta^{i}\left(b_{i}\right) \mathbb{D}_{a^{+}}^{\alpha} \phi^{i}\left(b_{i}^{-}\right) & =0, \quad \text { in }(0, T), i=m+1, \ldots, n, \\
\phi^{i}(T) & =0, \quad \text { in }\left(a, b_{i}\right), i=1, \ldots, n .
\end{aligned}
$$

Proof. The proof comes similarly as in lemma 3.3. 


\subsection{Optimal boundary control on a star graph}

We now go back to the original formulation with inhomogeneous Dirichlet boundary conditions and rename $u_{i}=v_{i}, i=2, \ldots, n$. From the index it is then clear that the first $m-1$ controls $v_{i}$ are the Dirichlet controls, while the $v_{i}, i=m+1, \ldots, n$ are the Neumann controls. We now consider the following optimal control problem:

$$
\min _{v \in \mathcal{U}_{a d}} \mathcal{J}(y(v), v)
$$

where

$$
\mathcal{J}(y, v)=\frac{1}{2} \sum_{i=1}^{n} \int_{0}^{T} \int_{a}^{b_{i}}\left(y^{i}(v)-y_{d}^{i}\right)^{2} d x d t+\frac{\gamma}{2} \sum_{i=2}^{n} \int_{0}^{T} v_{i}^{2} d t
$$

$y=\left(y^{i}\right)$ satisfies (111), $y_{d}^{i} \in H, \gamma>0$ and $\mathcal{U}_{a d}$ is closed convex of $\mathbb{R} \times \mathbb{R}_{+}$.

Theorem 4.2. There exists a unique solution $u \in \mathcal{U}_{a d}$ of the optimal control problem (155).

Proof. Let $\left\{v_{n}:=v_{i, n}, i=2, \ldots, n\right\} \subset \mathcal{U}_{a d}$ be a minimizing sequence such that

$$
\lim _{k \rightarrow+\infty} \mathcal{J}\left(y_{n}\left(v_{n}\right), v_{n}\right)=\min _{v \in \mathcal{U}_{a d}} \mathcal{J}(y(v), v) .
$$

Then there exists $C>0$ independent of $n$ such that

$$
\left\|v_{n}\right\| \leq C
$$

and observing that the control $v_{n}$ is associated to the state $y_{n}^{i}, i=2, \ldots, n$ solution of

$$
\begin{array}{lll}
\partial_{t} y_{n}^{i}+\mathcal{D}_{b_{i}^{-}}^{\alpha}\left(\beta^{i} \mathbb{D}_{a^{+}}^{\alpha} y_{n}^{i}\right)+q^{i} y_{n}^{i} & =f^{i}, \text { in }\left(a, b_{i}\right) \times(0, T), i=1, \ldots, n, \\
I_{a^{+}}^{1-\alpha} y_{n}^{i}\left(a^{+}\right) & =I_{a^{+}}^{1-\alpha} y_{n}^{j}\left(a^{+}\right) \text {in }(0, T), i \neq j=1, \ldots, n, \\
\sum_{i=1}^{n} \beta^{i}(a) \mathbb{D}_{a^{+}}^{\alpha} y_{n}^{i}\left(a^{+}\right) & =0, \quad \text { in }(0, T), \\
I_{a^{+}}^{1-\alpha} y_{n}^{1}\left(b_{1}^{-}\right) & =0, \quad \text { in }(0, T), \\
I_{a^{+}}^{1-\alpha} y_{n}^{i}\left(b_{i}^{-}\right) & =v_{i, n}, \quad \text { in }(0, T), i=2, \ldots, m \\
\beta^{i}\left(b_{i}\right) \mathbb{D}_{a^{+}}^{\alpha} y_{n}^{i}\left(b_{i}^{-}\right) & =v_{i, n}, \quad \text { in }(0, T), i=m+1, \ldots, n, \\
y_{n}^{i}(0) & =y^{0, i}, \quad \text { in }\left(a, b_{i}\right), i=1, \ldots, n .
\end{array}
$$

It follows from (151) and (152) that

$$
\left\|y_{n}^{i}\right\|_{L^{2}(0, T ; \mathbb{V})} \leq C
$$

and

$$
\left\|\partial_{t} y_{n}^{i}\right\|_{L^{2}\left(0, T ; \mathbb{V}^{*}\right)} \leq C .
$$

From (157), (159), (160) and the fact that $\mathcal{U}_{a d}$ is a closed convex subset of $\mathbb{R}^{n-1} \times \mathbb{R}_{+}$, we have that there exists $u \in \mathcal{U}_{a d}, y \in L^{2}(0, T ; \mathbb{V})$ and $\eta=\partial_{t} y \in L^{2}\left(0, T ; \mathbb{V}^{*}\right)$ such that

$$
\begin{aligned}
v_{n} & \rightarrow u \text { in } \mathcal{U}_{a d}, \\
y_{n} & \rightarrow y \text { weakly in } L^{2}(0, T ; \mathbb{V}), \\
\partial_{t} y_{n} & \rightarrow \partial_{t} y \text { weakly in } L^{2}\left(0, T ; \mathbb{V}^{*}\right)
\end{aligned}
$$


Using standard argument, (161),(162), (163) and (158), we prove that the control $u$ is associate to $y$, solution of (111) with $v=u$. It then follows from the semi continuity of the functional $\mathcal{J}$ that

$$
\mathcal{J}(y(u), u) \leq \frac{1}{2} \sum_{i=1}^{n} \int_{0}^{T} \int_{a}^{b_{i}}\left(y^{i}(u)-y_{d}^{i}\right)^{2} d x d t+\frac{\gamma}{2} \sum_{i=2}^{n} \int_{0}^{T} u^{2} d t \leq \liminf _{v \in \mathcal{U}_{a d}} \mathcal{J}\left(v_{n}\right)=\min _{v \in \mathcal{U}_{a d}} \mathcal{J}(v) .
$$

This means that

$$
\min _{v \in \mathcal{U}_{a d}} \mathcal{J}(y(v), v)=\mathcal{J}(y(u), u)
$$

The uniqueness of the control optimal $u$ follows from the strict convexity of $\mathcal{J}$.

The following theorem gives the sufficient optimality conditions.

Theorem 4.3. Let $u \in \mathcal{U}_{a d}$ be the optimal control solution of problem (155). Then there exists $p \in \mathbb{V}$ such that $(u, y=y(u), p)$ satisfies

$$
\begin{array}{ll}
\partial_{t} y^{i}+\mathcal{D}_{b_{i}^{-}}^{\alpha}\left(\beta^{i} \mathbb{D}_{a^{+}}^{\alpha} y^{i}\right)+q^{i} y^{i} & =f^{i}, \text { in }\left(a, b_{i}\right) \times(0, T), i=1, \ldots, n, \\
I_{a^{+}}^{1-\alpha} y^{i}\left(a^{+}\right) & =I_{a^{+}}^{1-\alpha} y^{j}\left(a^{+}\right) \text {in }(0, T), i \neq j=1, \ldots, n, \\
\sum_{i=1}^{n} \beta^{i}(a) \mathbb{D}_{a^{+}}^{\alpha} y^{i}\left(a^{+}\right) & =0, \quad \text { in }(0, T), \\
I_{a^{+}}^{1-\alpha} y^{1}\left(b_{1}^{-}\right) & =0, \quad \text { in }(0, T), \\
I_{a^{+}}^{1-\alpha} y^{i}\left(b_{i}^{-}\right) & =u_{i}, \quad \text { in }(0, T), i=2, \ldots, m \\
\beta^{i}\left(b_{i}\right) \mathbb{D}_{a^{+}}^{\alpha} y^{i}\left(b_{i}^{-}\right) & =u_{i}, \quad \text { in }(0, T), i=m+1, \ldots, n, \\
y^{i}(0) & =y^{0, i}, \quad \text { in }\left(a, b_{i}\right), i=1, \ldots, n . \\
-\partial_{t} p^{i}+\mathcal{D}_{b_{i}^{-}}^{\alpha}\left(\beta^{i} \mathbb{D}_{a^{+}}^{\alpha} p^{i}\right)+q^{i} p^{i} & =\sum_{i=1}^{n}\left(y^{i}-y_{d}^{i}\right), \text { in }\left(a, b_{i}\right) \times(0, T), i=1, \ldots, n, \\
I_{a^{+}}^{1-\alpha} p^{i}\left(a^{+}\right) & =I_{a^{+}}^{1-\alpha} p^{j}\left(a^{+}\right), \text {in }(0, T), i \neq j=1, \ldots, n, \\
\sum_{i=1}^{n} \beta^{i}(a) \mathbb{D}_{a^{+}}^{\alpha} p^{i}\left(a^{+}\right) & =0, \quad \text { in }(0, T), \\
I_{a^{+}}^{1-\alpha} p^{i}\left(b_{i}^{-}\right) & =0, \quad \text { in }(0, T) i=1, \ldots, m, \\
\beta^{i}\left(b_{i}\right) \mathbb{D}_{a^{+}}^{\alpha} p^{i}\left(b_{i}^{-}\right) & =0, \quad \text { in }(0, T) i=m+1, \ldots, n, \\
p^{i}(T) & =0, \quad \text { in }\left(a, b_{i}\right), i=1, \ldots, n .
\end{array}
$$

and

$$
\begin{aligned}
& \sum_{i=2}^{m} \int_{0}^{T}\left[\gamma u_{i}-\left(\beta^{i} \mathbb{D}_{a^{+}}^{\alpha} p^{i}\right)\left(b_{i}^{-}\right)\right] v_{i} d t \geq 0 \quad \forall v \in \mathcal{U}_{a d} . \\
& \sum_{i=m+1}^{n} \int_{0}^{T}\left[\gamma u_{i}+\left(I_{a^{+}}^{1-\alpha} p^{i}\right)\left(b_{i}^{-}\right)\right] v_{i} d t \geq 0 \quad \forall v \in \mathcal{U}_{a d}
\end{aligned}
$$

Proof. Equation (164) comes directly from the previous theorem. To prove (165) and (166), we take directional derivative with respect to $u$ in the direction $v$. We have

$$
\lim _{\lambda \rightarrow 0} \frac{\mathcal{J}(y, u+\lambda v)-\mathcal{J}(y, u)}{\lambda} \geq 0 \quad \forall v \in \mathcal{U}_{a d}
$$


which after a short calculation gives

$$
\sum_{i=1}^{n} \int_{0}^{T} \int_{a}^{b_{i}} z^{i}\left(y^{i}(u)-y_{d}^{i}\right) d x d t+\gamma \sum_{i=1}^{n} \int_{0}^{T} u_{i} v_{i} d t \geq 0 \quad \forall v \in \mathcal{U}_{a d}
$$

where $z^{i}$ is solmution of

$$
\begin{array}{ll}
\partial_{t} z^{i}+\mathcal{D}_{b_{i}^{-}}^{\alpha}\left(\beta^{i} \mathbb{D}_{a^{+}}^{\alpha} z^{i}\right)+q^{i} z^{i} & =0, \text { in }\left(a, b_{i}\right) \times(0, T), i=1, \ldots, n, \\
I_{a^{+}}^{1-\alpha} z^{i}\left(a^{+}\right) & =I_{a^{+}}^{1-\alpha} z^{j}\left(a^{+}\right) \text {in }(0, T), i \neq j=1, \ldots, n, \\
\sum_{i=1}^{n} \beta^{i}(a) \mathbb{D}_{a^{+}}^{\alpha} z^{i}\left(a^{+}\right) & =0, \quad \text { in }(0, T), \\
I_{a^{+}}^{1-\alpha} z^{1}\left(b_{1}^{-}\right) & =0, \quad \text { in }(0, T), \\
I_{a^{+}}^{1-\alpha} z^{i}\left(b_{i}^{-}\right) & =v_{i}, \quad \text { in }(0, T), i=2, \ldots, m \\
\beta^{i}\left(b_{i}\right) \mathbb{D}_{a^{+}}^{\alpha} z^{i}\left(b_{i}^{-}\right) & =v_{i}, \quad \text { in }(0, T), i=m+1, \ldots, n, \\
z^{i}(0) & =0, \quad \text { in }\left(a, b_{i}\right), i=1, \ldots, n .
\end{array}
$$

Let $p$ be regular function, multipying the first equation in (170) by $p$ and integrating by part over $\left(a, b_{i}\right) \times$ $(0, T)$, we get after some interpretation

$$
\sum_{i=m+1}^{n} \int_{0}^{T} v_{i} I_{a^{+}}^{1-\alpha} p^{i}\left(b_{i}^{-}\right) d t-\sum_{i=2}^{m} \int_{0}^{T} v_{i} \beta^{i}\left(b_{i}\right) \mathbb{D}_{a^{+}}^{\alpha} p^{i}\left(b_{i}^{-}\right) d t=\sum_{i=1}^{n} \int_{0}^{T} \int_{a}^{b_{i}} z^{i}\left(y^{i}-y_{d}^{i}\right) d x d t
$$

and the adjoint state equation

$$
\begin{array}{ll}
-\partial_{t} p^{i}+\mathcal{D}_{b_{i}^{-}}^{\alpha}\left(\beta^{i} \mathbb{D}_{a^{+}}^{\alpha} p^{i}\right)+q^{i} p^{i} & =\sum_{i=1}^{n}\left(y^{i}-y_{d}^{i}\right), \text { in }\left(a, b_{i}\right) \times(0, T), i=1, \ldots, n, \\
I_{a^{+}}^{1-\alpha} p^{i}\left(a^{+}\right) & =I_{a^{+}}^{1-\alpha} p^{j}\left(a^{+}\right), \text {in }(0, T), i \neq j=1, \ldots, n, \\
\sum_{i=1}^{n} \beta^{i}(a) \mathbb{D}_{a^{+}}^{\alpha} p^{i}\left(a^{+}\right) & =0, \quad \text { in }(0, T), \\
I_{a^{+}}^{1-\alpha} p^{i}\left(b_{i}^{-}\right) & =0, \quad \text { in }(0, T) i=1, \ldots, m, \\
\beta^{i}\left(b_{i}\right) \mathbb{D}_{a^{+}}^{\alpha} p^{i}\left(b_{i}^{-}\right) & =0, \quad \text { in }(0, T) i=m+1, \ldots, n, \\
p^{i}(T) & =0, \quad \text { in }\left(a, b_{i}\right), i=1, \ldots, n .
\end{array}
$$

Since $\sum_{i=1}^{n}\left(y^{i}-y_{d}^{i}\right) \in L^{2}(0, T ; H)$, equation (172) has a unique solution according to lemma 4.1. Conbining (169) and (171) we obtain

$$
\sum_{i=m+1}^{n} \int_{0}^{T} v_{i} I_{a^{+}}^{1-\alpha} p^{i}\left(b_{i}^{-}\right) d t-\sum_{i=2}^{m} \int_{0}^{T} v_{i} \beta^{i}\left(b_{i}\right) \mathbb{D}_{a^{+}}^{\alpha} p^{i}\left(b_{i}^{-}\right) d t+\gamma \sum_{i=1}^{n} \int_{0}^{T} u_{i} v_{i} d t \geq 0 \quad \forall v \in \mathcal{U}_{a d}
$$

that is

$$
\begin{aligned}
& \sum_{i=2}^{m} \int_{0}^{T}\left[\gamma u_{i}-\left(\beta^{i} \mathbb{D}_{a^{+}}^{\alpha} p^{i}\right)\left(b_{i}^{-}\right)\right] v_{i} d t \geq 0 \quad \forall v \in \mathcal{U}_{a d} \\
& \sum_{i=m+1}^{n} \int_{0}^{T}\left[\gamma u_{i}+\left(I_{a^{+}}^{1-\alpha} p^{i}\right)\left(b_{i}^{-}\right)\right] v_{i} d t \geq 0 \quad \forall v \in \mathcal{U}_{a d}
\end{aligned}
$$




\section{5. conclusion}

We investigated an optimal control of fractional diffusion Sturm-Liouville problem on a star graph where Sturm-Liouville operator is obtained as a composition of a right fractional Caputo derivative and a left fractional Riemann-Liouville derivative. The same results can be obtained if we consider a composition of a right fractional and a left fractional Riemann-Liouville derivative or a composition of a right fractional and a left fractional Caputo derivative. It suffices for that to well defined the domain of the operator.

\section{References}

[1] G. Mophou, G. Leugering and P. S. Fotsing, Optimal control of a fractional Sturm-Liouville problem on a star graph, Optimization, 2020

[2] A. Zettl, Sturm-Liouville Theory, in: Mathematical Surveys and Monographs, vol. 121, Americam Mathematical Society, 2005.

[3] G. Lumer, Connecting of local operators and evolution equations on a network, in: Lect. Notes Math., vol. 787, 1980, pp. 219-234.

[4] F. Ali Mehmeti. Nonlinear waves in networks. Mathematical Research, 80,Akademie-Verlag, Berlin, 1994.

[5] U. Brauer and G. Leugering. On boundary observability estimates for semidiscretizations of a dynamic network of elastic strings. Recent advances in control of PDEs. Control \&Cybernetics, 28:421-447, 1999.

[6] R. Dáger. Observation and control of vibrations in tree-shaped networks of strings. SIAM J. Control Optim., 43:590623, 2004.

[7] G. Leugering. Reverberation analysis and control of networks of elastic strings.Control of PDE and appl., Lect. Notes in Pure and Applied Math., pages 193-206, 1996.

[8] E. J. P. G. Schimdt. On the modelling and exact controllability of networks of vibrating strings. SIAM J. Control. Opt., 30:229-245, 1992.

[9] J. Von Below, Sturm-Liouville eigenvalue problems on networks, Math. Methods Appl. Sci. 10 (1988) 383-395.

[10] G. Leugering and G. Mophou. Instantaneous optimal control of friction dominated flow in a gas-network. BirkäuserVerlag: Proceedings of DFG-AIMS Workshop on Shape Optimization, Homogenization and Control. AIMS Senegal, Mbour, Senegal. 2017.

[11] D. Idczak and S. Walczak. Fractional sobolev space via Riemann-Liouville derivatives. Journal of Functional Spaces and Applications. Vol 2013, Article ID 128043, 15 pages.

[12] M. Klimek, T. Odzijewicz and A. B. Malinowska. Variational methods for the Sturm-Liouville problem. J. Math. Anal. Appl. 416 (2014) 402-426

[13] Y. Liu, S. Chen and L. Ou Solvability of Sturm-Liouville boundary value problems for multiple order fractional differential equations. Arab. J. Math Sci 22(2016) 207-231.

[14] N. Tokmagambetov and B. T. Torebek. Fractional analogue Sturm-Liouville operator. Documenta Mathematica 21(2016) 1503-1514.

[15] M. Dehghan and A. B. Mingarelli. FRactional Sturm-Liouville eigenvalue problem, I. arXiv preprint arXiv:1712.09891, 2017 -arxiv.org

[16] M. Klimek, O.P. Agrawal, Fractional Sturm-Liouville problem, Computers and Mathematics with Applications 66(2013) 795-812. 
[17] M. Klimek, O.P. Agrawal, On a regular fractional Sturm-Liouville problem with derivatives of order in $(0,1)$, in: Proceedings of the 13th Intermational Carpathian Control Conference, Vysoke Tatry (Podbanske), Slovakia, 28-31 May 2012. http://dx.doi.org/10.1109/CarpathianCC.2012.6228655.

[18] O.Kh. Abdullaev and J.R. Khujakulov On a problem for the time-fractional diffusion equation on a metric graphs. Uzbek Mathematical Journal, 2017, No 4.

[19] V. Mehandiratta et al., Existence and uniqueness results for a nonlinear Caputo fractional boundary value problem on a star graph J. Math. Anal. Appl., Vol. 447 (2019) pp. 1243-1264.

[20] K.B. Oldham and J. Spanier. The Fractional Calculus. Academic Press, New York, 1974.

[21] S.G. Samko, A.A. Kilbas and O. I. Marichev. Fractional integral and derivatives: Theory and applications. Gordon and Breach Science Publishers, Switzerland, 1993.

[22] A.A. Kilbas, H.M. Srivastava and J.J. Trujillo. Theory and Applications of Fractional Differential Equations. Elsevier Science B.V., Amsterdam, 2006.

[23] I.Podlubny. Fractional Differential Equations, Academic Press, San Diego, 1999.

[24] H.Brezis. Analyse fonctionnelle.Théorie et application. Masson(1983).

[25] J.L.Lions and E.Magenes. Problèmes aux limites non homogènes et applications. Paris, Dunod,1968,Vol. 1 et 2.

[26] J. L. Lions and M. Magenes, Problèmes aux limites non homogènes et applications. Dunod, vol.1 et 2, Paris 168. Zb1 0165.10801 MR 1159093.

[27] L.C. Evans, Partial Differential Equations, American Mathematical Society, Providence, Rhode Island, 1998.

[28] A. Zettl, Sturm-Liouville Theory, in: Mathematical Surveys and Monographs, vol. 121, Americam Mathematical Society, 2005.

[29] L. Debnath, D. Bhatta, Integral Transforms and their Applications, second ed., Chapman \& Hall/CRC, Taylor \& Francis Group, New York, 2007.

[30] R.K. Gupta, S.D. Gupta, Operational calculus of spheroidal wave angle function (generalized Legendre transform), Indian J. Pure Appl. Math. 8(1976) 602-610.

[31] R.K. Gupta, S.D. Gupta, Some operational properties of generalized Legendre transform and their applications II, Indian J. Pure Appl. Math. 8(1976) 589-601.

[32] G. Lumer, Connecting of local operators and evolution equations on a network, in: Lect. Notes Math., vol. 787, 1980, pp. 219-234.

[33] B.S. Pavlov, M.D. Faddeev, Model of free electrons and the scattering problem, Teoret. Mat. Fiz. 55 (1983) 257-269.

[34] J. Von Below, Sturm-Liouville eigenvalue problems on networks, Math. Methods Appl. Sci. 10 (1988) 383-395.

[35] M.G. Zagorodnii, Yu.V. Pokornyi, On the spectrum of second-order boundary-value problems on spatial networks, Uspekhi Mat. Nauk 44 (1989) 220-221.

[36] M. Klimek, O.P. Agrawal, Fractional Sturm-Liouville problem, Computers and Mathematics with Applications 66(2013) 795-812.

[37] M. Klimek, O.P. Agrawal, On a regular fractional Sturm-Liouville problem with derivatives of order in $(0,1)$, in: Proceedings of the 13th Intermational Carpathian Control Conference, Vysoke Tatry (Podbanske), Slovakia, 28-31 May 2012. http://dx.doi.org/10.1109/CarpathianCC.2012.6228655.

[38] J. Qi, S. Chen, Eigenvalue problems of the model from nonlocal continuum mechanics, J. Math. Phys. 52(073516)(2011).

[39] T.M. Atanackovic, B. Stankovic, Generalized wave equation in nonlocal elasticity, Acta Mech. 208(2009)1-10. 
[40] M. d'Ovidio, From Sturm-Liouville problems to fractional and anomalous diffusions, Stochastic Process. Appl. 122(2012) 3513-3544.

[41] John R. Graef, L. Kong, M. Wang, Existence and uniqueness of solutions for a fractional boundary value problem on a graph, Fract. Calc. Appl. Anal. 17 (2014) 499-510.

[42] V. Mehandiratta, M. Mehra and G. Leugering, Fractional optimal control problems an a star graph: optimality system and numerical solution. Mathematical control and related fields, Vol. 11 (1) (2021) pp. 189-209.

[43] V. Mehandiratta, M. Mehra and G. Leugering, A differnce scheme for the time-fractional diffusion equation on a metric star graph, Applied Numerical Mathematics, 158, (2020), 152-163.

[44] W. Zhang, W. Liu, Existence and Ulam's type stability results for a class of fractional boundary value problems on a star graph. Mathematical Methods in the Applied Sciences, (2020), https://doi.org/10.1002/mma.6516

[45] V. Mehandiratta, M. Mehra and G. Leugering, An approch based on Haar wavelet for the approximation of fractional calculus with application to initial and boundary value problems, Mathematical Methods in the Applied Sciences, (2020), https://doi.org/10.1002/mma.6800

[46] N. Kumar, M. Mehra, Legendre wavelet collocation method for fractional optimal control problems with fractional Bolza cost, Numerical methods for partial differential equations, (2020), https://doi.org/10.1002/num.22604

[47] N. Kumar, M. Mehra, Collocation method for solving non-linear fractional optimal control problems by using Hermite scaling function with error estimates, Optimal Control, Applications and Methods, (2020), https://doi.org/10.1002/oca.2681 